\section{Triterpenoids manipulate a broad range of virus-host fusion via wrapping the HR2 domain prevalent in viral envelopes}

\author{
Longlong $\mathrm{Si}^{1}$, Kun Meng ${ }^{1}$, Zhenyu Tian ${ }^{1}$, Jiaqi Sun ${ }^{1}$, Huiqiang Li ${ }^{1}$, Ziwei Zhang ${ }^{1}$, \\ Veronica Soloveva ${ }^{2}$, Haiwei $\mathrm{Li}^{1}$, Ge Fu ${ }^{1}$, Qing Xia ${ }^{1}$, Sulong Xiao ${ }^{1}$, Lihe Zhang ${ }^{1}$, Demin Zhou ${ }^{1}$ *
}

A trimer-of-hairpins motif has been identified in triggering virus-cell fusion within a variety of viral envelopes. Chemically manipulating such a motif represents current repertoire of viral fusion inhibitors. Here, we report that triterpenoids, a class of natural products, antagonize this trimer-of-hairpins via its constitutive heptad repeat-2 (HR2), a prevalent $\alpha$-helical coil in class I viral fusion proteins. Triterpenoids inhibit the entry of Ebola, Marburg, HIV, and influenza A viruses with distinct structure-activity relationships. Specifically, triterpenoid probes capture the viral envelope via photocrosslinking HR2. Profiling the Ebola HR2-triterpenoid interactions using amino acid substitution, surface plasmon resonance, and nuclear magnetic resonance revealed six residues accessible to triterpenoids, leading to wrapping of the hydrophobic helix and blocking of the HR1-HR2 interaction critical in the trimer-of-hairpins formation. This finding was also observed in the envelopes of HIV and influenza A viruses and might potentially extend to a broader variety of viruses, providing a mechanistic insight into triterpenoid-mediated modulation of viral fusion.

\section{INTRODUCTION}

Virus-host fusion is executed by viral envelopes via constituent fusion proteins during the life cycle of enveloped viruses $(1,2)$. Viral fusion proteins usually consist of two subunits: a host-membrane-binding subunit and a viral membrane-anchoring subunit (3). Recent work has identified three distinct classes of viral fusion proteins, which belong to evolutionarily distant viruses and exhibit limited sequence homology, along with at least four different mechanisms by which viral fusion-competent conformational changes are triggered. In addition, viral fusion proteins contain different types of fusion peptides and vary in their reliance on accessory proteins, further increasing the diversity of fusion proteins (3). Despite this staggering diversity, all characterized viral fusion proteins are found to mediate host-virus membrane fusion by transitioning from a variety of pre-fusion states to a membrane-embedded homotrimeric prehairpin intermediate and then to a post-fusion trimer-of-hairpins conformation, which brings the viral and host membranes into close proximity and thereby facilitates their union (Fig. 1A) (3). The dynamic process of forming the trimer-of-hairpins represents a potential target for therapeutic intervention against viral infections. However, identifying an exposed region as a targetable site remains a challenge due to heavy glycan shielding, sequence diversity, and multiple conformations.

Triterpenes, a class of secondary plant metabolites characterized by hydrophobic pentacyclic scaffolds, are found in a wide variety of folk medicines and possess valuable pharmacological properties. In particular, triterpenoids have been generally considered to be part of plant defense systems against pathogens and herbivores, although the molecular mechanism remains to be elucidated (4). Cell-based

\footnotetext{
${ }^{1}$ State Key Laboratory of Natural and Biomimetic Drugs, School of Pharmaceutical Sciences, Peking University, 38 Xueyuan Road, Beijing 100191, China. ${ }^{2}$ U.S. Army Medical Research Institute of Infectious Diseases, Fort Detrick, MD 21702, USA.

*Corresponding author. Email: deminzhou@bjmu.edu.cn
}

studies have confirmed that oleanolic acid (OA) and betulinic acid (BA), a lupane-type triterpene, show inhibitory activity against HIV infection; the BA analog bevirimat (PA-457) exhibited promising efficacy in clinical trials $(5,6)$. Very recently, analogs/derivatives of OA and echinocystic acid (EA), an OA-type triterpene, were found to exert a variety of inhibitory activities, strongly related to their structures, against influenza virus and hepatitis $\mathrm{C}$ virus (HCV) infection $(7,8)$. Further investigations suggested that these triterpenoid leads might bind to viral fusion proteins, including hemagglutinin (HA2) of influenza, E2 of HCV, and GP41 of HIV, thereby disrupting viral entry into the host cells (7-9). Whether the observed antiviral aspects of triterpenoids extend to other viruses and translate into a shared mechanism that can chemically antagonize the fusion of viruses with cellular membranes remains a topic of ongoing investigation.

Here, we report triterpenoids as a class of entry inhibitors of Ebola virus (EBOV) and Marburg virus (MARV), expanding the list of viruses for which triterpenoids act as entry inhibitors. We identified heptad repeat-2 (HR2), the prevalent heptad repeat sequence comprising an $\alpha$-helical coil in viral fusion proteins $(10,11)$, as a site accessible to triterpenoids that antagonize EBOV-cell fusion. Furthermore, as a critical element of the trimer-of-hairpins, HR2 was identified as a common target in the triterpenoid-mediated inhibition on viral entry of influenza virus and HIV, potentially extending to an increasingly diverse array of viruses. This finding provides mechanistic insights into the triterpenoid-HR2 complex as a switch in the modulation of virus-cell fusion, expanding the current repertoire of antiviral agents.

\section{RESULTS}

\section{Identification of triterpenoids as inhibitors by} EBOV entry assay

Inspired by the key role of triterpenoids in inhibiting the entry of HIV, HCV, and influenza virus and their strong structure-activity 


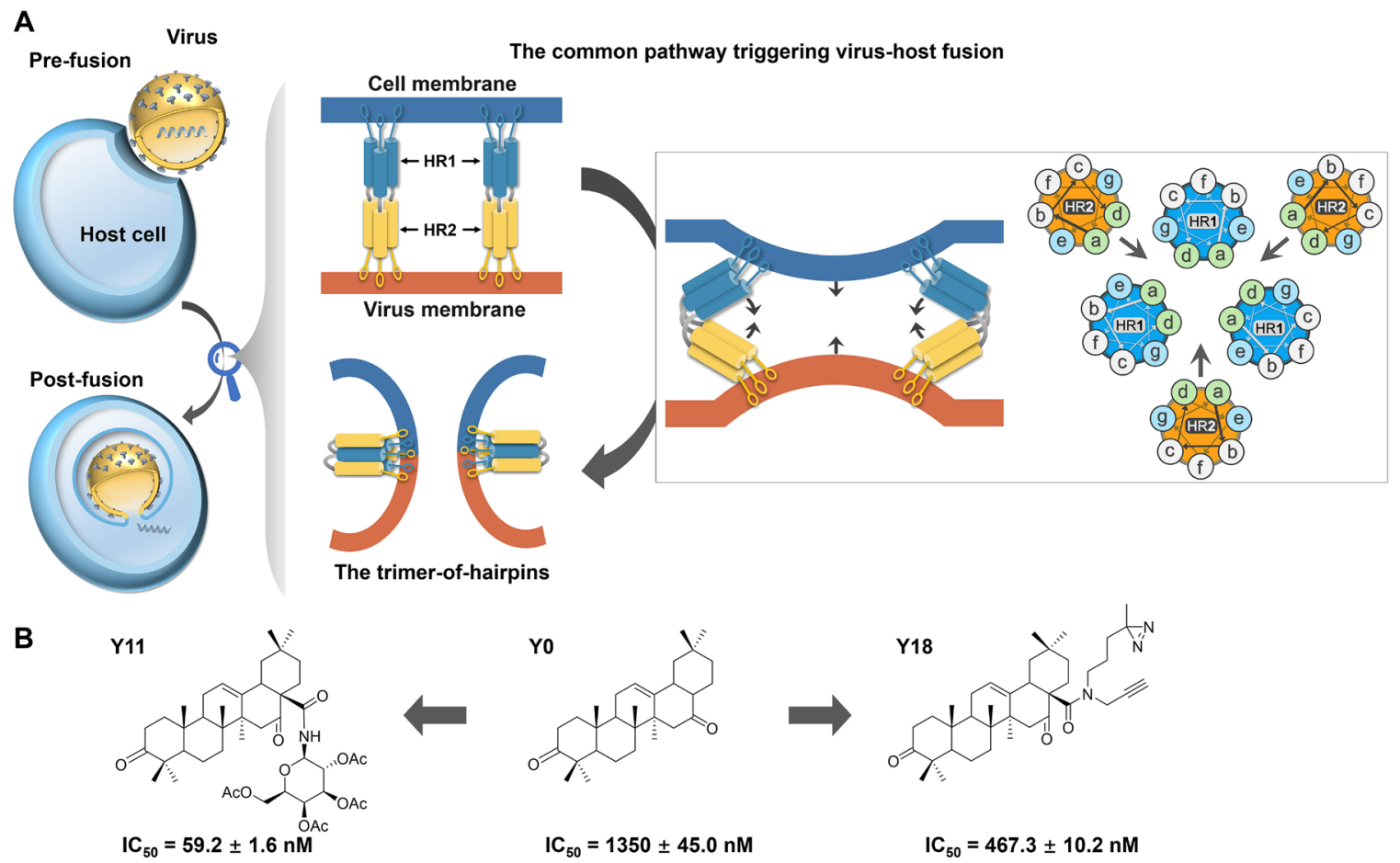

Fig. 1. Schematic representation of virus-host membrane fusion via a common trimer-of-hairpins by which EBOV, HIV, IAV, and other viruses with envelopes enter cells. (A) Pathway for converting distinct viral envelopes into the common trimer-of-hairpins conformation, which brings the viral and host membranes into close proximity for fusion. The interaction of HR2 with HR1 via hydrophobic residues, mainly at positions a and d, plays a critical role in the formation of the trimer-of-hairpins. (B) Lead compound and probe discovered among triterpenoid products that can inhibit EBOV-host fusion. 293T and A549 cells were used as the host cells.

relationship, we speculated on whether the entry of EBOVs could also be inhibited by triterpenoids. To alleviate safety concerns, we used Ebola pseudoparticles (EBOVpp), that is, Ebola glycoprotein (GP) assembled onto retroviral core particles, to screen a triterpenoid library consisting of hundreds of chemicals. Vesicular stomatitis virus (VSV) GP and WSN influenza envelope HA/neuraminidase (NA) protein pseudoparticles were tested in parallel to exclude toxic and nonspecific effects $(7,8)$. We identified a panel of triterpenoid compounds that inhibited the EBOVpp to different extents, strongly related to their structures, but did not inhibit VSV and WSN pseudoparticles (Fig. 1B and fig. S1, A and B). Specifically, Y11 and Y13, analogs of EA and OA, respectively, conjugated with acetylated galactose (7), displayed remarkable potencies: The $\mathrm{IC}_{50}$ (median inhibitory concentration) value for Y11 against the EBOV pseudotyped viruses was approximately $50 \mathrm{nM}$ for all five EBOV species tested in A549 and 293T cells (Fig. 1B, fig. S1A, and table S1). Furthermore, analyses of the strong structure-activity relationship revealed that a 3-keto group in the triterpenoid backbone and an acetyl substitution in the conjugated galactose are required for maintaining high anti-EBOV activity (fig. S1A). In addition, we found that Y11 inhibited the propagation of native EBOV (Makona) in HeLa cells [multiplicity of infection $(\mathrm{MOI})=1.5]$ and human foreskin fibroblast (HFF) cells (MOI $=20$ ): The $\mathrm{IC}_{50}$ values were 0.46 and $0.99 \mu \mathrm{M}$, and the $\mathrm{IC}_{90}$ values were 7.57 and $16.1 \mu \mathrm{M}$, respectively (fig. $\mathrm{S} 1 \mathrm{C}$ ); these values were comparable to those of the previously reported EBOV inhibitor 3.47 , which was used as a control $(12,13)$. The potency variation of $Y 11$ against native EBOV versus EBOVpp was potentially due to their multiple-round versus single-round turnover infection characteristics.

\section{EBOV fusion inhibition by triterpenoid lead Y11 via binding viral particles}

EBOV entry can further divided into two major steps: virus attachment to host cell receptor and virus-cell membrane fusion. Entry inhibitors can act at any stage of the entry process. A series of timeof-addition experiments $(7,8)$ were performed in parallel to determine the target stage of EBOV entry that is blocked by the triterpenoid lead Y11 (fig. S2A). We found that the addition of Y11 during EBOV-cell fusion stage had a much greater inhibitory effect than during EBOV attachment or post-entry stage but had a similar inhibitory effect to that of $\mathbf{Y 1 1}$ addition during the whole entry stage (fig. S2B), suggesting that $\mathrm{Y11}$ is acting during EBOV fusion stage.

We further addressed the binding of Y11 with EBOVpp directly by conducting two experiments in parallel, namely, pretreatment of the cells and pretreatment of the virus assay (fig. S2A). We found that the pretreatment of EBOVpp rather than of cells with Y11 led to substantial viral entry inhibition (fig. S2B). This result is markedly different from that of E-64d, which inhibits EBOV entry into cells by targeting the host cysteine protease (13). This effect suggested that Y11 targets EBOVpp particles rather than cells by which Y11 inhibits EBOV fusion.

\section{Identification of the envelope subunit HR2 as the target site of triterpenoid probes}

As the viral envelope was the only difference between EBOVpp and the VSV and WSN pseudoviruses (14), the specific anti-EBOV activity of the triterpenoid leads, along with the time-of-addition studies, indirectly suggests that the triterpenoid leads bind to EBOV GP. To directly capture the target site of the triterpenoid lead 
compounds, Y18, a triterpenoid probe containing dual-functional chemical moieties, namely, a diazirine (15) and an alkyne (16) substitution at 28-COOH, was synthesized and tested for its anti-EBOV activity and action mechanism (Fig. 1B). The diazirine and alkyne moieties were introduced for photocrosslinking of the target proteins, followed by click conjugation with biotin to capture and enrich the cross-linked protein (Fig. 2A). It was found that Y18 exerted potent inhibitory activity on EBOV fusion by the same action mechanism as that of Y11 (Fig. 1B, figs. S1B and S2, and table S1). Incubation of Y18 with the crude protein extracts from the pseudovirusproducing cells followed by ultraviolet (UV) activation specifically cross-linked EBOV GP, which was precipitated by an azido-biotin resin in Western blot analysis but not VSVG or influenza A virus (IAV) HA (Fig. 2, A and B). This specific cross-linking was prevented by the competitive compounds Y0 and Y11, as demonstrated in a parallel experiment, but not by the inactive analog Y12 (Fig. 2, A and B), confirming the direct interaction of GP with Y18. To identify the targeting site within EBOV GP, the purified GP protein was used for incubation with Y18, followed by UV activation. The Y18/GP photocrosslinked complex was subjected to highresolution mass spectrometry (MS), and a 573.41819 increase in molecular weight, corresponding to photoactivated Y18, was detected in the peptide 623-IDQIIHDFVDK-633 (Fig. 2C and fig. S3A), which is known as HR2 on the viral fusion protein $(17,18)$. The cross-linking site within the peptide was localized to I627 (Fig. 2C), a constitutive residue of HR2, by the matrix-assisted laser desorption/ ionization MS (MALDI-MS) analysis of tryptic digests. This result suggested that the EBOV envelope subunit HR2 is the target domain, with $\mathrm{I} 627$ as an accessible residue.

\section{Verification of the specific affinity between HR2 and triterpenoid leads by SPR}

To confirm the interaction between triterpenoids and HR2 identified by chemical probes and photocrosslinking-MS experiments, we then tested whether the triterpenoids had high affinity to HR2 using the surface plasmon resonance (SPR) assay. The N-trimer (N39) and C-terminal GP2 peptides (fig. S3A), representing HR1 and HR2, respectively, as previously reported (19), were immobilized separately on a CM5 chip with the tested compounds flowing across their surface. We found that Y11 and Y18 bound to HR2, exhibiting a strong dose-dependent response, with respective $K_{\mathrm{D}}$ (equilibrium dissociation constant) values of 5.4 and $16.8 \mu \mathrm{M}$ (Fig. 2D); no specific binding to HR1 or a randomized version of HR2 peptide (613HDWTKNITD-621) was found for Y11 and Y18 in the parallel experiment (fig. S3, B and C). The inactive analog Y12 and the unrelated compound E-64d, the EBOV entry inhibitor targeting the host cysteine protease (13), did not bind to either immobilized peptide (Fig. $2 \mathrm{D}$ and fig. S3, B and C). In addition, the interaction between HR1 and HR2 was significantly reduced in the presence of Y11: The $K_{\mathrm{D}}$ value increased from 1.0 to $23.4 \mu \mathrm{M}$ (Fig. 2E and fig. S3D); however, this interaction was not reduced by either Y12 or E-64d (Fig. 2E and fig. S3D). These results suggested that the triterpenoid leads had a specific affinity to HR2, and thus interfered the HR1-HR2 interaction, by which the formation of the trimer-of-hairpins could be blocked.

\section{Nuclear magnetic resonance characterization of HR2 residues interacting with the triterpenoid leads}

We then elucidated the global spatial interactions between HR2 and the triterpenoid leads by nuclear magnetic resonance (NMR) (Fig. 3 and fig. S4) (20). The addition of Y11 to an HR2 peptide solution (fig. S4, A and B) was found to induce chemical shift changes for the acylamino groups of residues I623, D624, Q625, I626, I627, D629, and F630 but not H628 (Fig. 3A), indicating that H628 is irrelevant for triterpenoid binding. Specifically, Y11 was revealed by NMR TOCSY (total correlation spectroscopy) to contact I627 and F630, as the signals of $\mathrm{I} 627 \beta-\mathrm{H}$ and $\mathrm{F} 630 \alpha-\mathrm{H}$ disappeared upon the addition of Y11 (Fig. 3B). Furthermore, the nuclear Overhauser effect (NOE) signals between Q625 $\beta-\mathrm{H}$ and Y11 25-H and between $\mathrm{I} 627 \alpha-\mathrm{H}$ and Y11 Gal acetyl-H (Fig. 3C and fig. S4C), determined by NMR ROESY (rotating frame Overhauser effect spectroscopy), demonstrated the proximity of these groups and demonstrated the orientation of the triterpenoid Y11 across the HR2 core surface to be head to head and tail to tail (Fig. 3D). Further elucidation of the NOE signal network between Y11 and HR2, mainly including I627 NH_Y11 19/30-H, I623 $\delta$-H_Y11 NH, D629 $\beta$-H_Y11 NH, I626 $\delta$-H_Y11 25-H, I623 $\gamma$-H_Y11 22-H, and I627 $\gamma$-H_Y11 18-H (Fig. 3C and fig. S4C), revealed more details of their spatial interactions (Fig. 3D). In contrast, the inactive analog Y12 did not induce any chemical shift change for the HR2 residues (fig. S4D), and no NOE signal was observed from the Y12-HR2 complex; furthermore, Y11 or Y12 had no effect on the chemical shift of HR1 or a randomized version of HR2 (fig. S4D), confirming the specificity of the binding of Y11 to HR2.

The interactions of residues of the HR2 core with Y11, elucidated by the NMR data, were reflected by the docking calculations (fig. S5) (7), showing that most hydrophobic residues were shielded by the triterpenoid hydrophobic scaffold (fig. S5A). On the basis of the minimum free energy analyses, the simulation data showed that the estimated binding energy and inhibition constant $\left(K_{\mathrm{i}}\right)$ were $-8.41 \mathrm{kcal} / \mathrm{mol}$ and $0.5 \mu \mathrm{M}$, respectively, for the Y11-HR2 complex and $-7.86 \mathrm{kcal} / \mathrm{mol}$ and $1.4 \mu \mathrm{M}$, respectively, for the Y18-HR2 complex (fig. S5D). In addition, the docking calculations revealed the mild discrepancy between the conformations of Y18 and Y11 in wrapping the HR2 coil: The replacement of the spatial Gal moiety (Y11) with a linear group (Y18) brought the triterpenoid 3=O closer to K622, leading to hydrogen bond formation between $3=\mathrm{O}$ of $\mathrm{Y} 18$ and the amido group of K622, while Q625 was more distant from the triterpenoid Y18 due to the lack of spatial Gal moiety (fig. S5A). This difference might explain why K622 was critical for Y18, while Q625 appeared to be irrelevant (fig. S5E), further verifying their head-to-head and tail-to-tail orientations and the global residue interactions. This was also supported by the observation that I627 in HR2 is photocrosslinked by the diazirine moiety in Y18 (Fig. 2C), reflecting their proximity. The docking model of Y11 and Y18 with HR2 was supported by the docking calculations with inactive analog Y12 and HR2 mutants: Y12 was not able to bind to the same site as that of Y11; the alanine mutation of HR2 residues induced the conformation changes of Y11 and Y18 (fig. S5, B and C), and much higher binding energy and $K_{\mathrm{i}}$ were observed in the HR2 alanine mutation docking models compared to the wild-type HR2 docking models (fig. S5D), suggesting that Y11 and Y18 had much less interactions with HR2 alanine mutants and much lower affinity to HR2 alanine mutants, consistent with the following biological mapping data (fig. S5E).

\section{Verification of $\mathrm{HR} 2$ residues that interact with triterpenoid leads by biological mapping}

We then used amino acid substitution mapping $(21,22)$ to biologically verify which residues of HR2 were potentially interacting with the triterpenoids (fig. S5E). We first performed alanine screening to 
A

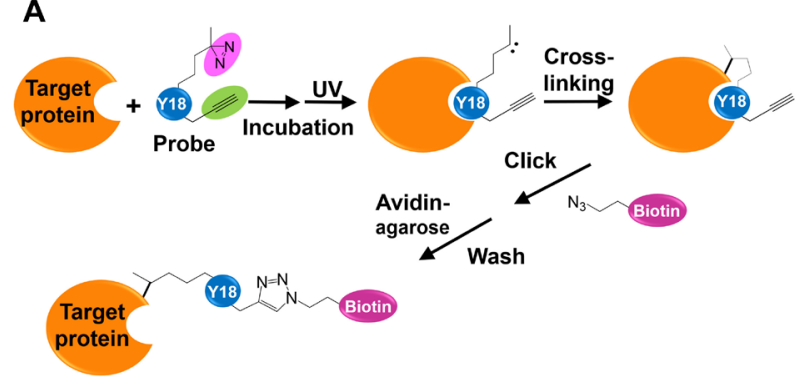

C

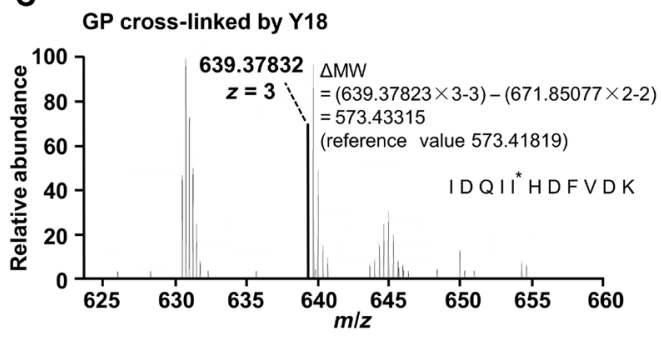

GP wild type
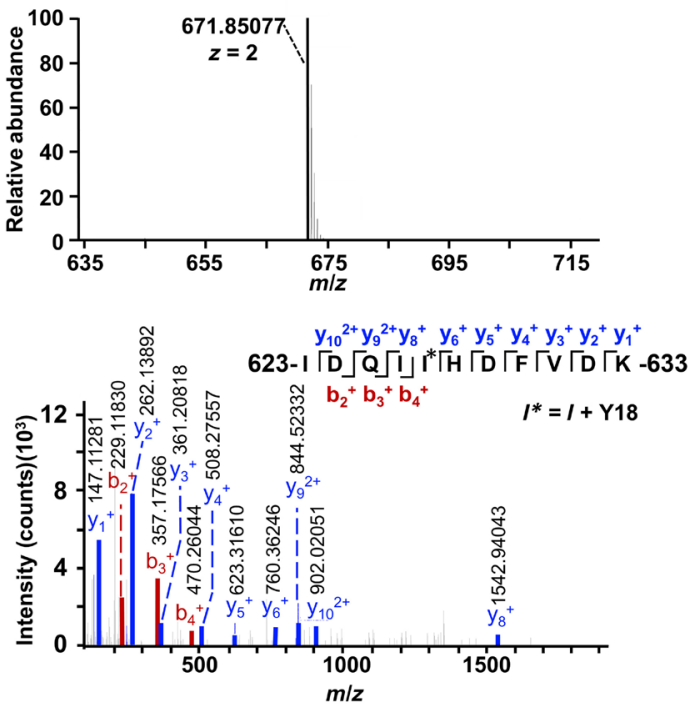

D
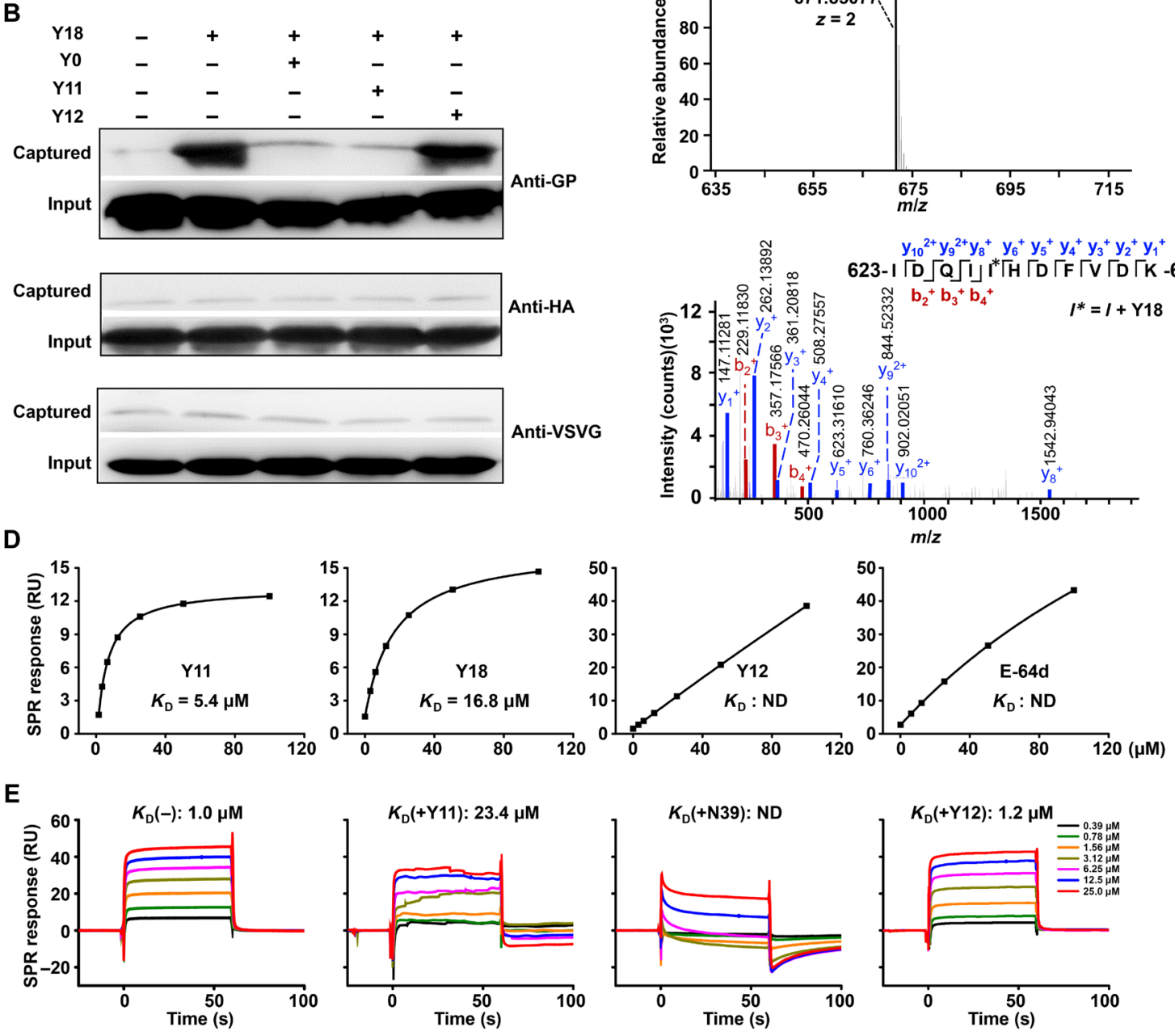

Fig. 2. Identification of the EBOV envelope GP as the target of the triterpenoid leads. (A) Scheme representing the mechanism by which the triterpenoid probe Y18 covalently captures the target protein via sequential photocrosslinking capture and click-mediated biotin harvest. (B) Western blot analyses of the cross-linked protein with Y18 using anti-GP, anti-HA, or anti-VSVG antibodies. The packaged cell lysates, pretreated with $50 \mu \mathrm{M} \mathrm{Y0,} \mathrm{Y11,} \mathrm{or} \mathrm{Y12} \mathrm{for} 30$ min, were incubated with $5 \mu \mathrm{M}$ Y18 and then exposed to UV light $(365 \mathrm{~nm}$ ) before being treated with azide-containing biotin. (C) Mass spectrum of the captured protein with the cross-linking site identified by peptide mapping. $m / z$, mass/charge ratio. (D) SPR characterization of the affinity between the triterpenoid compounds, $\mathbf{Y 1 1}$ and $\mathbf{Y 1 8}$, and the HR2 peptide, which was immobilized on an SA sensor chip. Y12 and E-64d served as negative controls. (E) SPR characterization of the effects of the triterpenoid compounds on HR1-HR2 interaction. HR2 was allowed to flow across the HR1 chip surface in the absence or presence of the lead compound; EbolZN39IQ and E-64d served as positive and negative controls, respectively.

evaluate the effect of each individual substitution on virus infectivity. The mutations of residues K622, D624, Q625, I627, H628, D629, and F630 resulted in the maintenance of 5 to $80 \%$ of parental infectivity, while less than $0.1 \%$ infectivity was detected for the mutations at residues I623 and I626 (fig. S5E). This result reflected the criticality of each constitutive residue of HR2 for maintaining infectivity, and the latter two residues were excluded from further exploration. Then, the potency of Y11 against each mutant virus was tested to determine the contribution of each residue. We found that K622A, D624A, Q625A, and D629A reduced the potency of Y11 by two- to fourfold (fig. S5E), reflecting their moderate contributions to the binding of HR2 to the triterpenoid lead. F630A and I627A reduced 
A
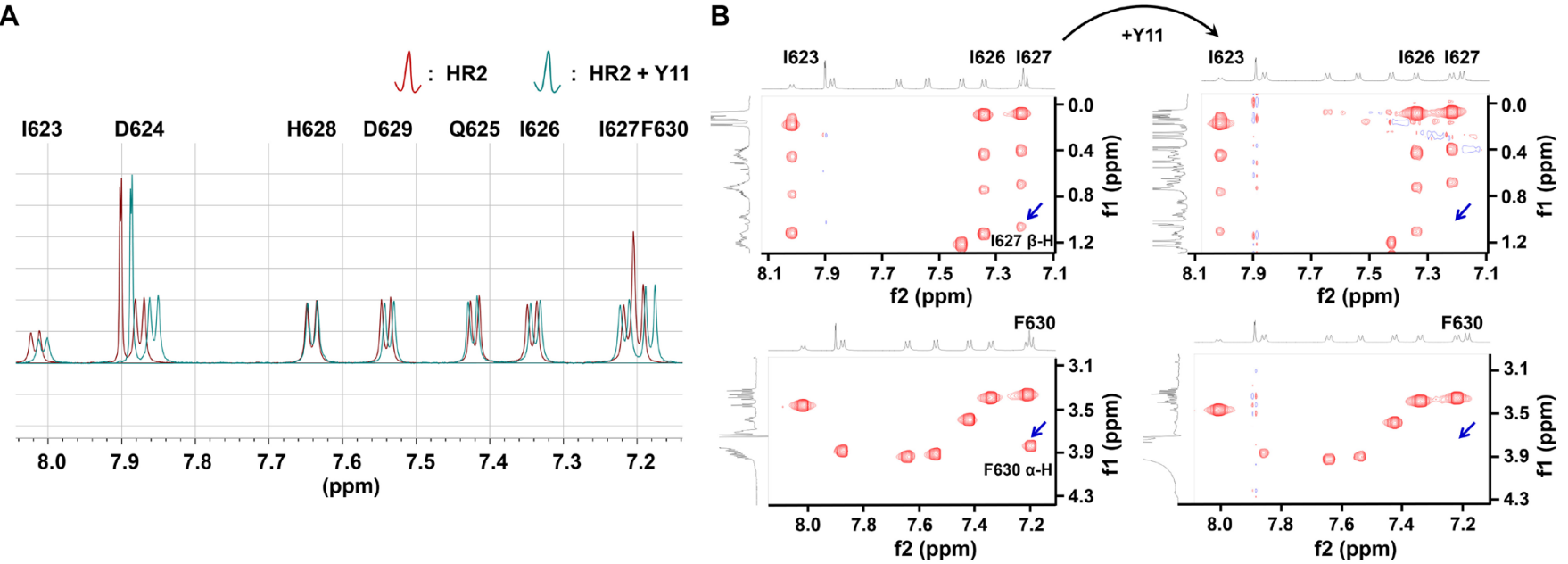

C

\begin{tabular}{|c|c|c|c|}
\hline \multirow{2}{*}{ No. } & \multirow{2}{*}{$\begin{array}{c}\text { NOE signals } \\
(7.22,0.26)\end{array}$} & \multicolumn{2}{|c|}{ Signal assignment } \\
\hline & & 1627_NH & Y11_29/30H \\
\hline 2 & $(7.06,0.17)$ & 1623_8H & Y11_NH \\
\hline 3 & $(7.06,1.93)$ & D629_ßH & Y11_NH \\
\hline 4 & $(3.34,3.28)$ & I627_aH & Y11_galH \\
\hline 5 & $(1.33,0.31)$ & Q625_pH & Y11_25H \\
\hline 6 & $(1.15,0.31)$ & 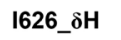 & Y11_25H \\
\hline 7 & $(0.48,1.43)$ & 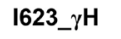 & Y11_22H \\
\hline 8 & $(0.42,2.52)$ & $1627 \_\gamma \mathrm{H}$ & Y11_18H \\
\hline
\end{tabular}

D

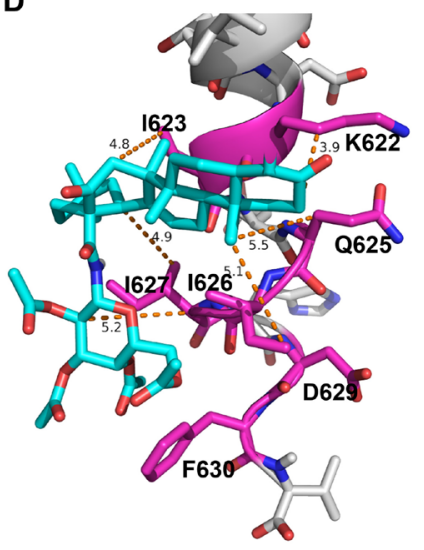

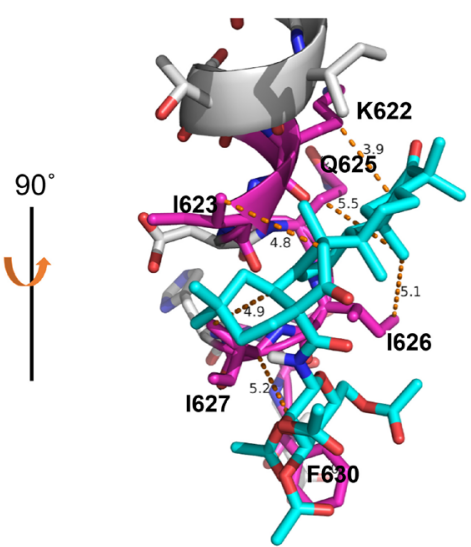

Fig. 3. Characterization of the spatial interactions within the triterpenoid-HR2 complex. (A) Overlapped HR2 acylamino ${ }^{1} \mathrm{H}$ NMR spectra in the absence and presence of Y11 to differentiate the residues interacting with the triterpenoid lead. (B) NMR TOCSY spectra of the HR2 peptide (KIDQIIHDF) in the absence or presence of Y11. Upon the addition of Y11 (ratio of HR2/Y11 as 1:1), the signals (blue arrows) of $1627 \beta-\mathrm{H}$ and F630 $\alpha-\mathrm{H}$ from HR2 shifted. (C) Assignment of each of the intermolecular NOE signal from the triterpenoid-HR2 complex. (D) Global HR2-Y11 complex inferred from the NOE signals plus docking simulation (Protein Data Bank: 5JQ3). The intermolecular NOEs are indicated by orange dashed lines.

the potency of Y11 by approximately 7- and 10-fold (fig. S5E), respectively, revealing their significant contributions to binding the triterpenoid lead. In contrast, H628A appeared to have no effect on the potency of Y11, similar to the mapping of all residues for interaction with compound E-64d. This result indicated that H628, unlike other residues, does not bind the triterpenoid, potentially due to its distant location. Similar results were observed for Y18, except that K622A decreased the potency of Y18 by 10-fold, while Q625A did not affect the potency of Y18 (fig. S5E), suggesting that K622 was critical, while Q625 was irrelevant, for Y18 binding. This finding is consistent with the docking calculations showing that a hydrogen bond formed between 3=O of $\mathbf{Y 1 8}$ and the amino group of K622, while Q625 was more distant from Y18 (fig. S5A). These data further confirmed the molecular structure model of the interactions between the triterpenoid lead and HR2 (Fig. 3D).

\section{MARV entry inhibition by triterpenoid lead compounds Y11 and Y18}

Similar to EBOV, MARV is the other major member of the family Filoviridae. To explore whether the antiviral spectrum of triterpenoids can extend to other filoviral pathogens, we tested the activity of the triterpenoid lead compounds Y11 and Y18 against MARV using Marburg pseudoparticle (MARVpp) entry assay. We found that Y11 and $\mathrm{Y} 18$ were able to inhibit the infection of MARV: The $\mathrm{IC}_{50}$ values were 2.29 and $5.52 \mu \mathrm{M}$, respectively, and the $\mathrm{IC}_{90}$ values were 11.1 and $33.3 \mu \mathrm{M}$, respectively (table S1); these values were 10- to 50 -fold higher than those in EBOVpp models, indicating that Y11 and Y18 exhibited diminished activity against MARV compared to EBOV. These results were consistent with the following sequence alignment data.

The sequence alignment of MARV (strain Musoke) HR2 and EBOV HR2s indicated that four amino acid residues I623, Q625, I626, and D629 of MARV HR2 were conserved compared to EBOV; however, two key amino acid residues K622 and F630 of EBOV HR2s were mutated to Q and E, respectively, in MARV HR2 (fig. S3A). The mutations K622Q and F630E in MARV HR2 may be responsible for the diminished activity of $\mathbf{Y 1 1}$ and Y18 against MARV, consistent with the biological mapping data: K622A reduced the potency of Y11 and Y18 by approximately 3- and 10-fold, respectively; F630A reduced the potency of $\mathrm{Y} 11$ and $\mathrm{Y} 18$ by approximately 7 - and 22 -fold, respectively (fig. S5E).

Therefore, Y11 and Y18 can be considered as potential antivirals against filoviral pathogens, including EBOV and MARV. In addition, 
A

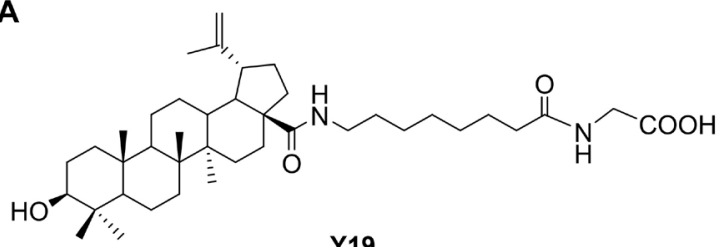

Y19

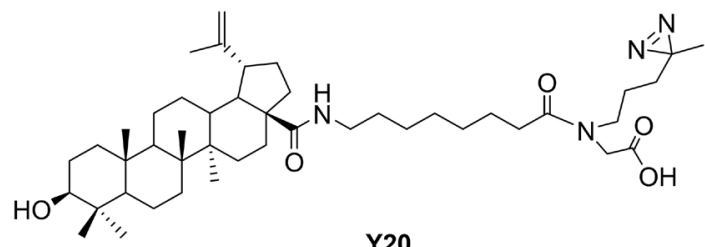

Y20

B

C

$y_{16}{ }^{+} y_{15}{ }^{+} y_{14}{ }^{+} y_{13}{ }^{+} y_{12}{ }^{+} y_{11}{ }^{2+} y_{10}{ }^{+} y_{9}{ }^{+} y_{8}{ }^{+} y_{7}{ }^{+} y_{6}{ }^{+} y_{5}{ }^{+} y_{4}{ }^{+} y_{3}{ }^{+} y_{1}{ }^{+}$

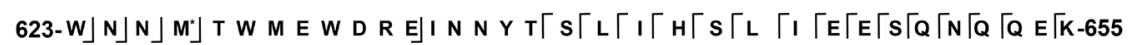

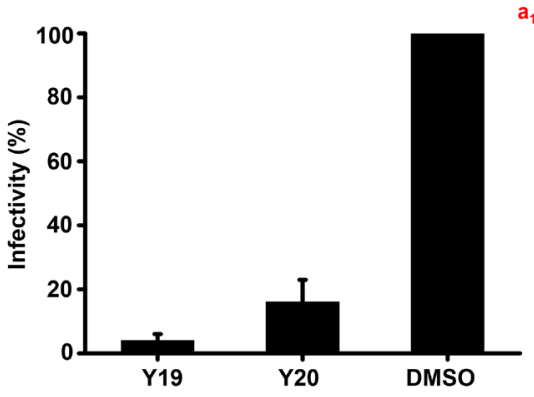
$\mathrm{a}_{1}{ }^{+} \mathrm{a}_{2}{ }^{2+} \mathrm{b}_{3}{ }^{2+} \mathrm{a}_{4}{ }^{+}$ $\mathrm{a}_{12}{ }^{2+}$
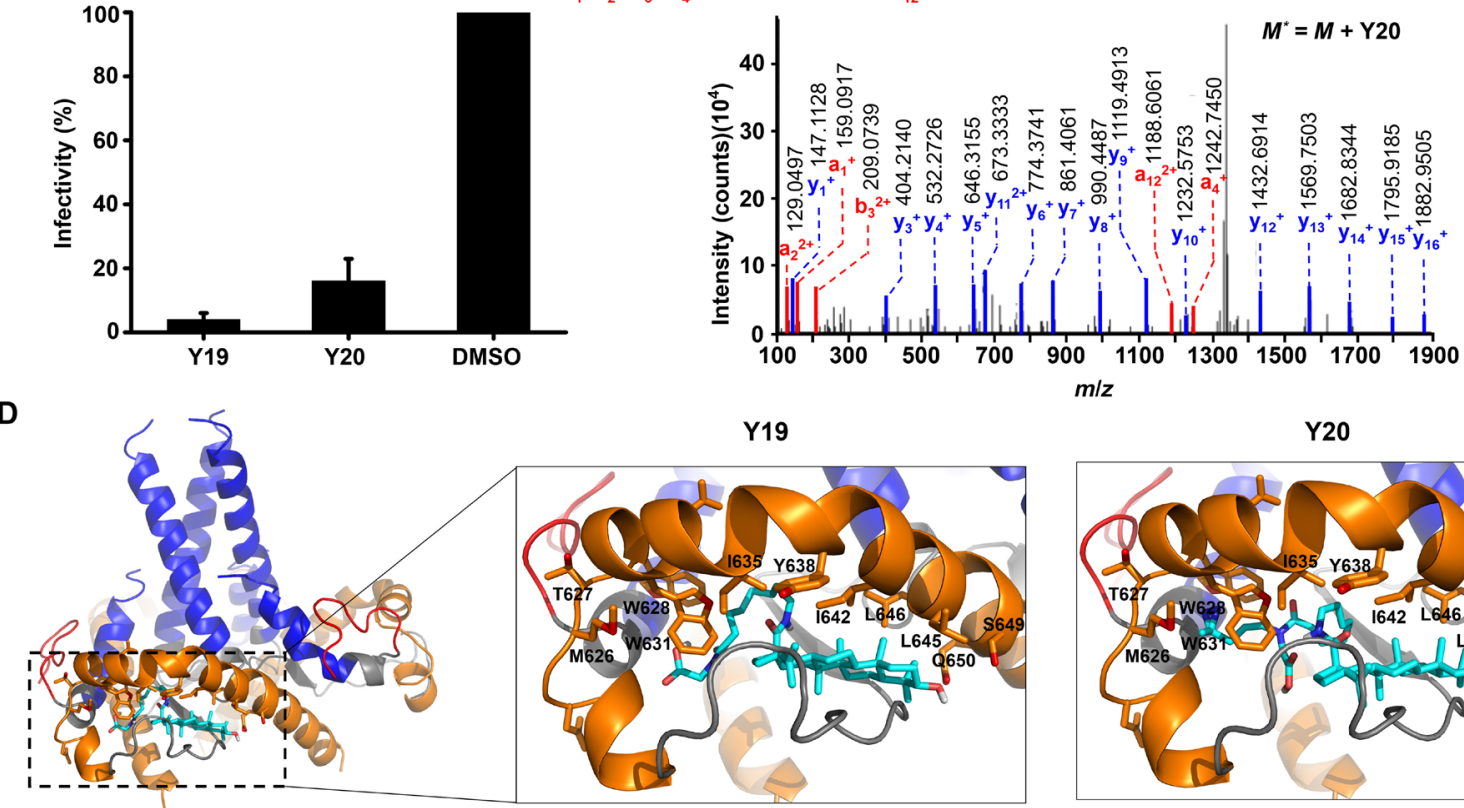

Y20

Fig. 4. Identification of HIV GP41 HR2 as the target domain of the triterpenoid lead compounds. (A) Structures of the anti-HIV triterpenoid lead Y19 and its photocrosslinking probe Y20. (B) Characterization of the inhibitory activity of the triterpenoids Y19 and Y20 on the infectivity of HIV-1 pseudovirions. The concentrations of Y19 and Y20 used were $1 \mu \mathrm{M}$. CEM 4 was used as the host cell. (C) Identification of HR2 as the photocrosslinked domain by MS. The HIV GP41 protein was photoaffinity labeled with the Y20 probe and then analyzed by peptide mapping. A molecular weight increase of 772.5598, corresponding to photoactivated Y20, was observed for the peptide 623-WNNMTWMEWDREINNYTSLIHSLIEESQNQQEK-655. The cross-linking site at M626 was confirmed by the MALDI-MS analysis of tryptic digests. (D) Structural representations of Y19 and Y20 binding to the HIV GP41 HR2 domain (Protein Data Bank: 4TVP), as inferred from docking simulations. The -COOH group of the lead compounds lies in the polar pocket generated by D624, N625, and T627, and the 3-OH group of the lead compounds lies near the polar residues S649 and Q650. The diazirine group of $\mathbf{Y 2 0}$ is in close proximity to M626, the residue that is cross-linked by Y20. Multiple hydrophobic interactions are observed between the lead compounds and their binding sites, namely, W628, W631, 1635, Y638, 1642, L645, and L646.

the potency of triterpenoid lead compounds against MARV could further increase when their structures were further optimized on the basis of the MARV HR2.

\section{Identification of HR2 as a targeting domain in the triterpenoid-mediated inhibition of HIV and influenza virus fusion}

In addition to EBOV and MARV, a variety of triterpenoids and their derivatives have been reported to inhibit the entry of many other distinct viruses; however, their targets and action mechanisms have not been verified by direct evidences so far. To explore whether the findings in the present Ebola study could be extended to other viruses, we explored the targets of triterpenoids in another two virus models, for example, HIV and influenza virus, using the photocrosslinking triterpenoid probes.

Similar to EBOV, HIV envelope protein GP120/GP41 mediates its entry into host cells (23). BA and its derivative Y19 (Fig. 4A) have been reported as entry inhibitors of HIV by interacting with HIV envelope protein (24); however, the target site has not been identified by direct evidence. To directly identify their target site, Y20 (Fig. 4A), a triterpenoid probe containing a diazirine moiety, was synthesized and tested for activity with its parent Y19 as a control compound. Both Y19 and Y20 exhibited inhibitory activity on the infectivity of HIV-1 pseudovirions (Fig. 4B). Using the same approach as in the exploration of EBOV GP, the incubation of Y20 with the HIV envelope protein followed by MS analysis of the UV-activated mixture revealed a molecular weight increase of 772.5598 , corresponding to the photoactivated Y20, in the peptide 623-WNNMTWMEWDREINNYTSLIHSLIEESQNQQEK-655, which contains HR2 in the HIV GP41 protein (Fig. 4C and fig. S6A) (25). The cross-linking site within the peptide was localized to M626 according to the MALDI-MS analysis of the tryptic digests (Fig. 4C). The SPR assays indicated that Y20 binds tightly to HIV-HR2 but not to HIV-HR1 $(9,26)$; both were separately immobilized on a CM5 
chip with the tested compounds flowing across its surface (fig. S6, B and $\mathrm{C}$ ). Also similar to the findings in the EBOV study, the addition of Y20 to HIV-HR1/HIV-HR2 mixtures significantly reduced their affinity (fig. S6D), by which the formation of the trimer-of-hairpins could be blocked. The docking simulations also indicated multiple hydrophobic interactions between the hydrophobic backbones of Y19 and Y20 and the residues that constitute HR2, namely, W628, W631, I635, Y638, I642, L645, and L646 [Protein Data Bank: 4TVP (25)] (Fig. 4D). The -COOH group of Y19 or Y20 lies in the polar pocket generated by D624, N625, and T627, and the 3-OH group of Y19 or Y20 lies near the polar residues S649 and Q650. In the above binding model, the diazirine group of $\mathbf{Y} 20$ is in close proximity to M626, the residue that is cross-linked by $\mathbf{Y 2 0}$, which was consistent with the photocrosslinking results (Fig. 4C), further confirming the binding orientation. We thus concluded that the HR2 in the HIV envelope protein GP41 is a target for the triterpenoid leads.

In the influenza virus model study, OA and its derivative Y3 (Fig. 5A) have been reported to have a significant inhibitory effect on influenza virus entry by binding influenza virus envelope protein HA (7). In addition, Y3 showed promising anti-influenza virus efficacy in mouse model (7). Similarly, to directly identify their target site, Y21 (Fig. 5A), a triterpenoid probe containing a diazirine group on the galactose moiety, was synthesized and tested for activity with its parent Y3 as a control compound (7). Both Y3 and Y21 exhibited inhibitory activity on the infectivity of influenza HA-HIV pseudovirions (Fig. 5B). Using the same approach as in the exploration of EBOV GP and HIV GP, the incubation of Y21 with HA protein followed by MS analysis of the UV-activated mixture revealed a molecular weight increase of 825.5027 , corresponding to photoactivated Y21, in the peptide 471-NNAKEIGNGCFEFYHK-486, which is known as HR2 in HA protein [influenza A/California/04/2009 (H1N1)] (Fig. 5C and fig. S7A) (27). The cross-linking site was localized to one of the residues from N471 to I476 based on the MALDI-MS analysis of the tryptic digests (Fig. 5C). The interaction between the influenza virus HR2 and the triterpenoid Y3 was further verified by mapping experiments (fig. S7B), which were consistent with the docking calculation that revealed the interactions of the hydrophobic backbones of the lead compounds Y3 and Y21 with HR2 [Protein Data Bank: 3LZG (28)], including residues V465, K466, Q468, L469, and N472 (Fig. 5D). A hydrogen bond was formed between the diazirine group of Y21 and N472, the potential cross-linking site within HR2, which was consistent with the photocrosslinking observations (Fig. 5C). Therefore, we concluded that HR2 is the direct target in the triterpenoid-mediated inhibition of influenza virus fusion. The results from the HIV and influenza virus model studies suggested that the findings in Ebola model can be shared by other types of triterpenoids and their respective viruses, at least including $\mathrm{BA}$ derivative and HIV, and OA derivative and influenza virus.

\section{DISCUSSION}

Viruses initiate infection by attaching to cell surface receptors and fusing viral and cell membranes. The membrane fusion is directed by viral fusion proteins. HR2 is a prevalent domain in class I viral fusion proteins, like HR1, and is characterized by a heptad repeat sequence designated as a, b, c, d, e, f, and g (Figs. 1A and 6A) $(29,30)$. As exceptional sequences, both HR1 and HR2 share dual hydrophobic residues at positions a and $\mathrm{d}$ and self-fold into $\alpha$-helical coils that interact with each other via hydrophobic regions at the protein surfaces, oligomerizing to form superhelical assemblies (1). This phenomenon has been observed in an increasing variety of viruses, including HIV (30), influenza virus (31), and EBOV (10), in which the N-terminal HR1 domain of the viral membrane-anchoring subunit forms a parallel, trimeric coiled coil onto which the C-terminal HR2 region coils, forming a trimer-of-hairpins and ultimately bringing the virus and host membranes into close proximity for fusion (Fig. 6). The tight van der Waals forces between the coils make the formation of the coiled coil thermodynamically favorable, rendering the identification of chemical antagonists to block the formation of the trimeric $\alpha$-helical coiled coil highly challenging. Thus far, only long complementary peptide sequences, such as T20 peptide, have been developed for the inhibition of the fusion of HIV or other viruses $(32,33)$. Here, we report the addition of EBOV and MARV to the list of viruses for which triterpenoids act as antagonists against viral and cell membrane fusion. Using the photocrosslinking probe, we directly captured the EBOV GP as the target of triterpenoid fusion inhibitors with HR2 as the binding site. Our finding suggested that HR2 might be an exposable domain in viral fusion proteins during virus-host fusion; this possibility was further supported by the EBOV HR2 peptide-mediated induction of antibodies capable of inhibiting EBOV entry, including all five EBOV subtypes (fig. S8). Combining our findings that HR2 in the viral fusion proteins of EBOV, HIV, and influenza virus is the target of their triterpenoid fusion inhibitors and the prevalence of the HR2 motif in a variety of viral envelopes, the triterpenoid-mediated inhibition of viral and cell membrane fusion via HR2 might be potentially extended to an increasing variety of viruses.

The HR2 motif is not usually considered a conceptual target for drug design due to its helicity; chemical ligands with high affinity and specificity have not been previously reported $(13,26,34)$. Triterpenoids, which provide a pentacyclic backbone for hydrophobic interactions with the six nonpolar residues of HR2, as determined by amino acid substitution, SPR, and NMR spectroscopy, can crosswrap HR2 in a putative head-to-head and tail-to-tail orientation. In particular, the hydrophobic regions of the coil surfaces composed of residues a and $\mathrm{d}$, which provide the thermodynamic driving force for the coiled coils and allow multiple $\alpha$-helical coils to wrap around each other, were shielded by triterpenoid leads (Figs. 3D, 4D, 5D, and 6). In addition, the high conservation of HR2 residues among different EBOV strains (fig. S3A), as demonstrated by the impaired infectivity phenotype of all HR2 mutants (fig. S5E), and the much lower glycan shielding than at other parts of the viral membrane (18) make HR2 an ideal target for disrupting the HR1-HR2 interaction. Despite the evolutionary distance among pathogenic viruses, such as the devastating EBOV, MARV, HIV, and IAV in this study, the structural homology of the trimer-of-hairpins, on the basis of experimentally determined three-dimensional (3D) structures, may translate into a shared mechanism with which hosts can use triterpenoids to antagonize membrane fusion in a variety of viruses. Overall, the combined factors of helix wrapping, hydrophobicity, and the terminal hydrogen bond give rise to a distinct HR2-triterpenoid molecular architecture.

Although the evolution of viruses remains controversial $(35,36)$, the high degree of structural similarity, including the HR1-HR2 interaction conserved among distinct viral fusion proteins and the similar trimer-of-hairpins causing the virus-host membrane fusion (3), implies that HR2-containing viral fusion proteins may have a common origin. The broad antiviral aspects of triterpenes, likely owing to the accessibility of hydrophobic HR2 to the pentacyclic 
A

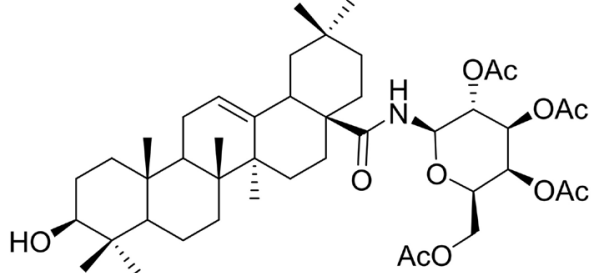

Y3

B

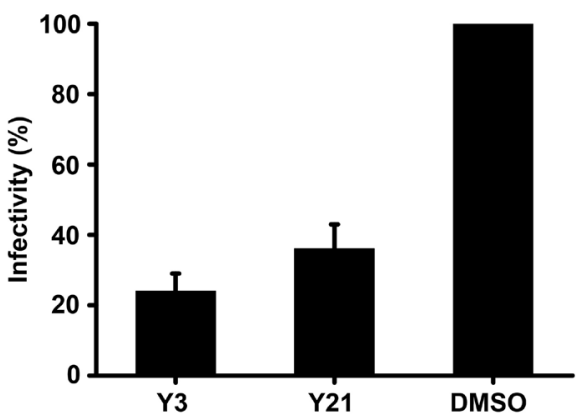

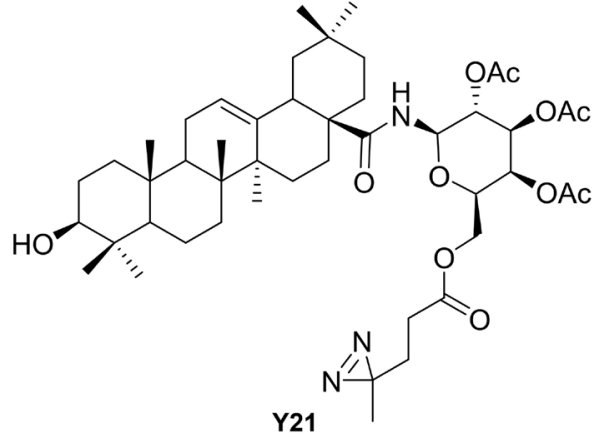

C
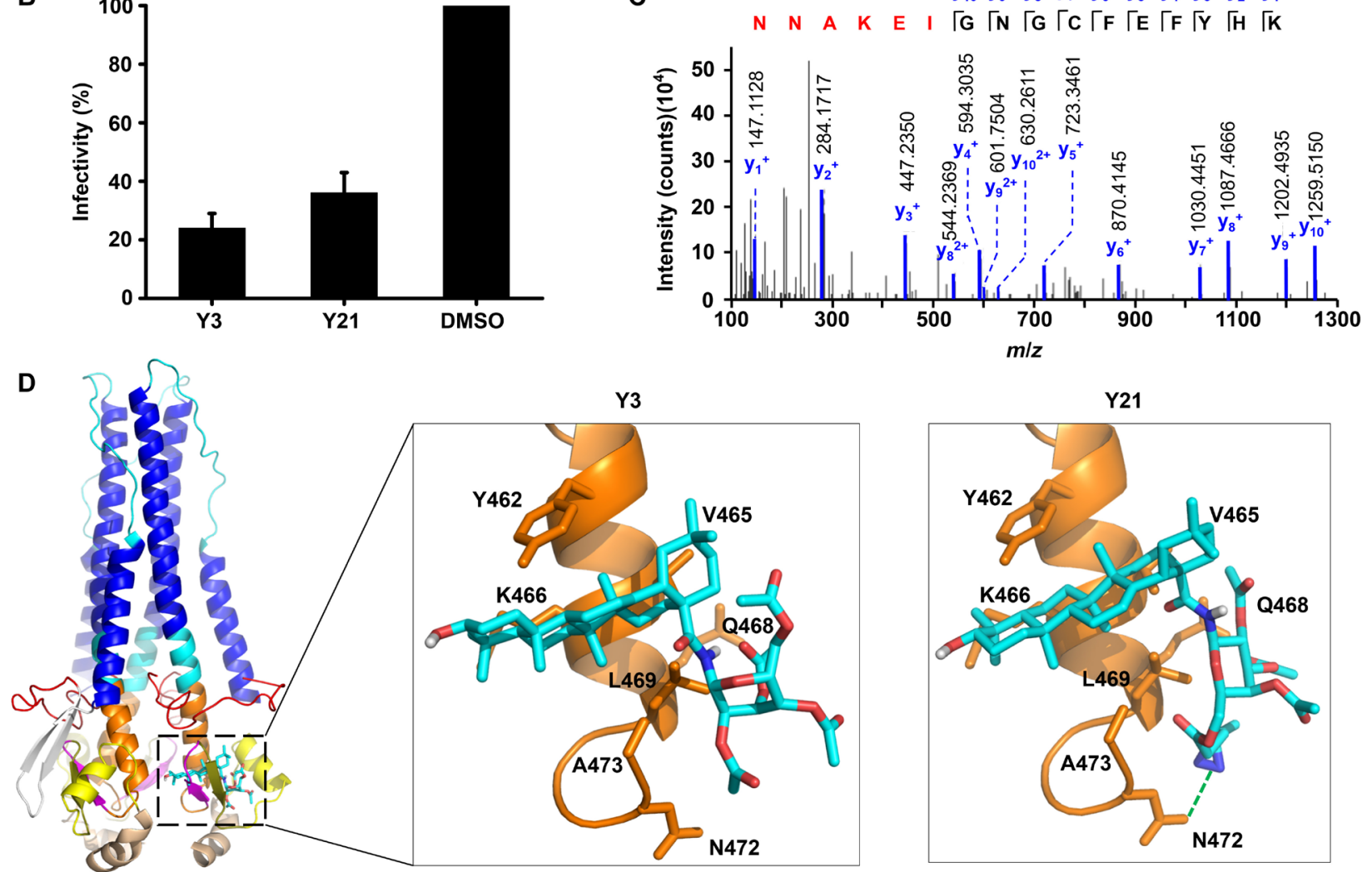

Fig. 5. Identification of HR2 in influenza HA2 as the target domain for triterpenoid leads. (A) Structures of the anti-influenza triterpenoid lead Y3 and its photocrosslinking probe Y21. (B) Characterization of the inhibitory activity of the triterpenoids Y3 and Y21 on the infectivity of influenza HA-HIV pseudovirions (IAVpp). A549 was used as the host cell. (C) Identification of HR2 as the photocrosslinked domain by MS. The influenza HA protein was photoaffinity labeled with the Y21 probe and then analyzed by peptide mapping. A molecular weight increase of 825.5027, corresponding to photoactivated Y21, was observed in the peptide 471-NNAKEIGNGCFEFYHK-486 [influenza A/California/04/2009 (H1N1)]. The cross-linking site was within a residue between N471 and 1476 based on the MALDI-MS analysis of tryptic digests. (D) Structural representations of Y3 and Y21 binding to the HR2 domain of influenza HA (Protein Data Bank: 3LZG) according to both the docking simulation and the alanine screening data. The amino acid residues that are involved in interactions with the lead compounds are labeled. The Y21 diazirine group forms a hydrogen bond with N672, which is located near the cross-linking site.

hydrophobic backbone, imply that triterpenes may be the native antagonists for their respective viruses (fig. S9). As demonstrated in this study, OA-based triterpenoids have a high propensity to bind $462-\mathrm{Y}_{(\mathrm{a})} \mathrm{E}_{(\mathrm{b})} \mathrm{K}_{(\mathrm{c})} \mathrm{V}_{(\mathrm{d})} \mathrm{K}_{(\mathrm{e})} \mathrm{S}_{(\mathrm{f})} \mathrm{Q}_{(\mathrm{g})} \mathrm{L}_{(\mathrm{a})} \mathrm{K}_{(\mathrm{b})} \mathrm{N}_{(\mathrm{c})} \mathrm{N}_{(\mathrm{d})} \mathrm{A}_{(\mathrm{e})}-473$, the HR2 sequence shared by influenza viruses; $\mathrm{BA}$-based triterpenoids tend to bind 627- $\mathrm{T}_{(\mathrm{g})} \mathrm{W}_{(\mathrm{a})} \mathrm{M}_{(\mathrm{b})} \mathrm{E}_{(\mathrm{c})} \mathrm{W}_{(\mathrm{d})} \mathrm{D}_{(\mathrm{e})} \mathrm{R}_{(\mathrm{f})} \mathrm{E}_{(\mathrm{g})} \mathrm{I}_{(\mathrm{a})} \mathrm{N}_{(\mathrm{b})} \mathrm{N}_{(\mathrm{c})} \mathrm{Y}_{(\mathrm{d})} \mathrm{T}_{(\mathrm{e})} \mathrm{S}_{(\mathrm{f})} \mathrm{L}_{(\mathrm{g})} \mathrm{I}_{(\mathrm{a})}$ $\mathrm{H}_{(\mathrm{b})} \mathrm{S}_{(\mathrm{c})} \mathrm{LI}_{(\mathrm{d})} \mathrm{E}_{(\mathrm{e})} \mathrm{ES}_{(\mathrm{a})} \mathrm{Q}_{(\mathrm{b})}-650$, an HR2 variant shared by HIV; and EA-based triterpenoids tend to bind $622-\mathrm{K}_{(\mathrm{g})} \mathrm{I}_{(\mathrm{a})} \mathrm{D}_{(\mathrm{b})} \mathrm{Q}_{(\mathrm{c})} \mathrm{I}_{(\mathrm{d})} \mathrm{I}_{(\mathrm{e})} \mathrm{H}_{(\mathrm{f})} \mathrm{D}_{(\mathrm{g})}$ $\mathrm{F}_{(\mathrm{a})}-630$, an HR2 variant shared by EBOV (Fig. 6A and fig. S9). Despite the lack of amino acid sequence conservation between differ- ent viruses, the residues at the same positions in HR2 have similar characteristics (Fig. 6A), giving rise to similar spatial structures, which provide the structural basis by which triterpenoids, as a chemical class, may act as broad-spectrum viral fusion antagonists.

The triterpenoid wrapping of HR2 has significant implications for the development of antiviral drugs. Unlike neutralizing antibodies and peptide fusion inhibitors that target long epitopes with multiple conformations, triterpenoids recognize relatively smaller domains and cross the whole surface of the HR2 coil, and thus, they can be developed as powerful fusion inhibitors. Currently, a series of triterpenoid 

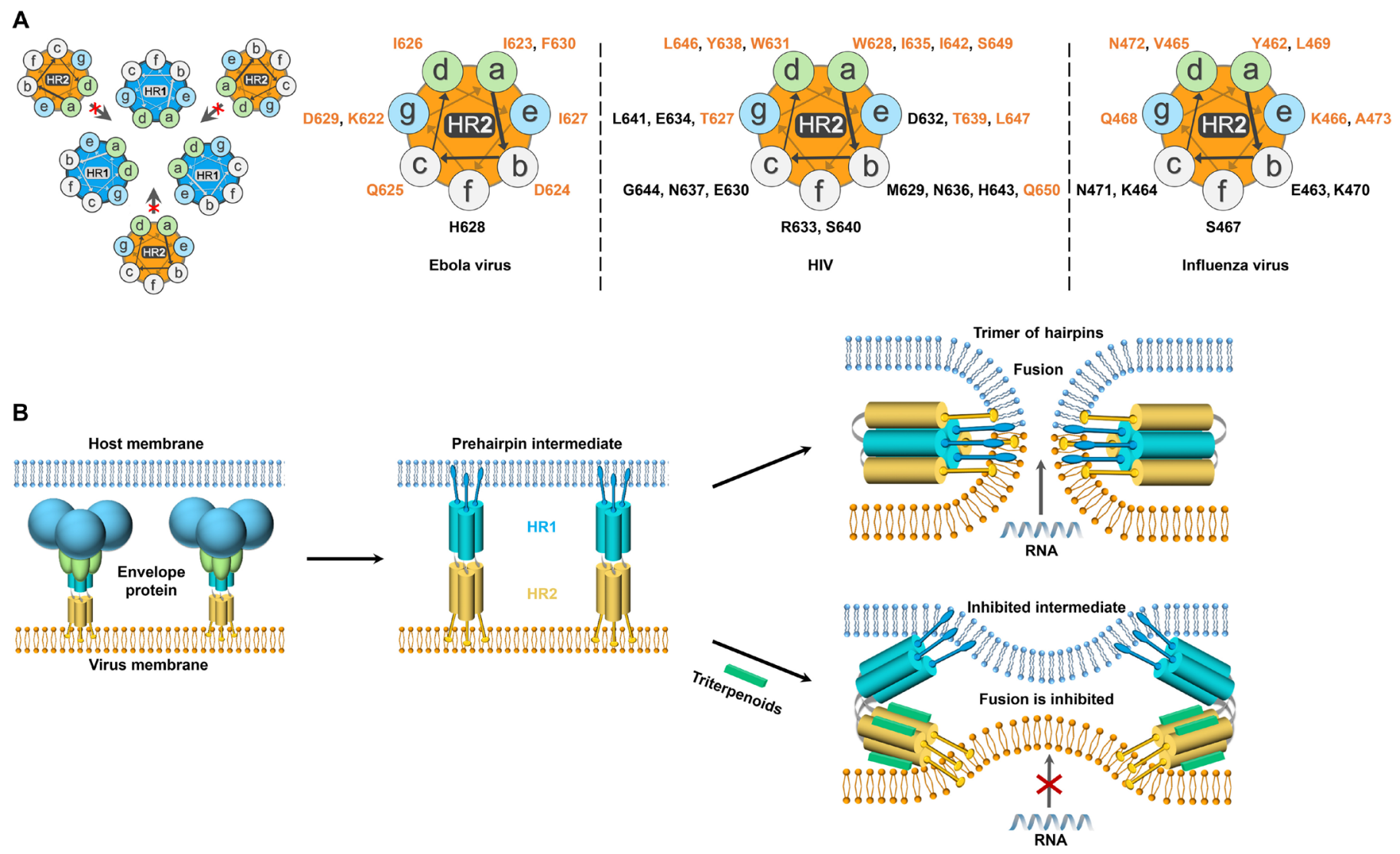

Fig. 6. The structural similarity of HR2 provides the basis by which triterpenoids block virus-cell membrane fusion. (A) Common helical wheel representation of the six-helix bundle, the post-fusion form of viral fusion proteins, and helical wheel representations of the HR2 domains of EBOV GP2, HIV GP41, and IAV HA, with the amino acid residues identified. The residues at positions a and $d$ are hydrophobic residues, whereas the residues at positions $b, c, e, f$, and $g$ are polar residues. The residues at positions a and $d$ of the HR1 domain form the trimer interface, and the HR2 domain folds into the groove of the HR1 trimer via its hydrophobic face formed by the $\mathrm{a}$ and $\mathrm{d}$ residues. The residues involved in triterpenoid binding (depicted in orange) are primarily distributed at positions a, $\mathrm{d}$, e, and g. (B) Shared mechanism for the triterpenoid-mediated inhibition of membrane fusion between cells and the EBOV, HIV, or influenza virus. The triterpenoids bind to the HR2 domain of a class I viral fusion protein, disrupting its interaction with the HR1 trimer. Thus, membrane fusion between the virus and the host cell is inhibited.

leads, including BA-, OA-, and EA-based chemicals targeting HIV, $\mathrm{HCV}$, influenza virus, EBOV, and MARV, are being explored. Notably, bevirimat (PA-457), a BA-based HIV inhibitor, has been developed in clinical trials as a maturation inhibitor and therefore deserves to be reconsidered as an anti-fusion antagonist for further development. Triterpenes, mainly consisting of the lupane, oleanane, and ursane types $(7,8)$, are secondary plant metabolites found in various plants, constituting up to $30 \%$ of dry weight in a few species (37). This provides a rich source of chemical diversity for a stock of deterrents to EBOV, MARV, HIV, and influenza virus and may potentially extend to a wider variety of viruses. Overall, the broad antiviral properties of triterpenoids and the identification of the prevalent HR2 coil as an exposable target pave the way for modulations of virus-host fusion that are of general significance, emerging as a new frontier in drug discovery and development.

\section{MATERIALS AND METHODS Cell lines}

Human embryonic kidney (HEK) 293T cells, Madin-Darby canine kidney (MDCK) cells, human lung adenocarcinoma A549 cells, HeLa cells, CEM 4 cells, and human HFF cells were obtained from the
American Type Culture Collection and grown in Dulbecco's modified Eagle's medium (Gibco BRL Inc., Gaithersburg, MD, USA) supplemented with $10 \%$ fetal bovine serum (PAA Laboratories, Linz, Austria) and cultured at $37^{\circ} \mathrm{C}$ with $5 \% \mathrm{CO}_{2}$.

\section{Reagents}

Rabbit anti-GP (Ebola/Zaire) polyclonal antibody was obtained from Immune Technology Corp. (New York, USA). Rabbit anti-HA (H1N1) monoclonal antibody was obtained from Sino Biological Inc. (Beijing, China). Mouse anti-VSVG monoclonal antibody, horseradish peroxidase (HRP)-conjugated anti-rabbit immunoglobulin $\mathrm{G}(\mathrm{IgG})$, and antimouse IgG were obtained from Sigma-Aldrich (St. Louis, MO, USA). E-64d (TCI Development Co. Ltd., Shanghai, China) and compound 3.47 were $>98 \%$ pure and were used as reference compounds. All tested compounds were dissolved in dimethyl sulfoxide (DMSO) to a stock concentration of $10 \mathrm{mM}$. The purity of all evaluated biological compounds was $>95 \%$, as determined by high-performance liquid chromatography (HPLC) (Agilent 1260) with a detection wavelength of $215 \mathrm{~nm}$.

\section{Plasmids}

Plasmids expressing wild-type EBOV GP were obtained by inserting GP complementary DNA (cDNA) from the corresponding EBOV 
subtype (Zaire, strain Mayinga 1976, GenBank accession number U23187.1; Sudan, strain Gulu, GenBank accession number YP 138523.1; Bundibugyo, strain Uganda 2007, GenBank accession number ACI28624.1; Ivory Coast, strain Cote d'Ivoire 1994, GenBank accession number ACI28632.1; and Reston, strain Siena 1992, GenBank accession number AAC54891.1) between the Kpn I and $\mathrm{Xba}$ I sites of the PCMV3 mammalian expression vector (Sino Biological Inc., Beijing, China). Plasmid expressing MARV envelope protein GP was obtained by inserting codon-optimized Make Victoria MARV GP cDNA (strain Musoke, GenBank accession number YP_001531156.1) between the Hind III and Xba I sites of the PCMV3 mammalian expression vector. Plasmids expressing the wild-type HIV-1 envelope protein were obtained by inserting HIV-1 gp160 cDNA (strain NDK) between the Kpn I and Xba I sites of the PCMV3 mammalian expression vector. The HA and NA genes were amplified from IAV strain A/WSN/33(H1N1) cDNA by polymerase chain reaction and then inserted between the Kpn I and Eco RI or Xho I sites, respectively, of the pcDNA4/TO vector (Invitrogen, Carlsbad, CA). Other plasmids included pCMV-VSVG (Addgene), pNL 4-3 (NIH AIDS Reagent Program), and pAdvantage (Promega). The EBOV GP and influenza virus HA mutants were constructed with the Site-Directed Mutagenesis Kit (Agilent Technologies) and confirmed by sequencing (BGI, Beijing). All plasmids used for transfection were amplified using the Maxiprep Kit (Promega) according to the manufacturer's instructions.

\section{Proteins}

The expression and purification of HIV gp41 protein (Ala517-Asn711, GenBank accession number P03377.1) were performed as described previously (38). Briefly, the gene encoding gp41 protein was synthesized by BGI (Beijing, China) and cloned into the pET21a (+) vector. $\mathrm{N}$-terminal methionine was added, and $\mathrm{Cys}^{603}$ and Cys ${ }^{609}$ were mutated to Ala. BL21 (DE3) was used as the host strain for gp41 expression. Expressed protein was extracted with SDS and purified by a combination of gel filtration and hydroxyapatite chromatographies. The protein was folded by exchanging SDS for Fos-Choline-12 (DPC; Affymetrix) and concentrated to $10 \mathrm{mg} / \mathrm{ml}$ with an Amicon-Ultra $15 \mathrm{ml}, 30-\mathrm{kDa}$ concentrator (EMD Millipore). The EBOV GP (catalog no. 40304-V08B1) and influenza virus HA (catalog no. 11055V08B) used for the photoaffinity labeling were purchased from Sino Biological Inc. As shown in the product specifications, the baculovirusinsect cell expression system was used to express the EBOV GP (Met1-Gln650, GenBank accession number AAC54887.1) and influenza virus HA (Met1-Gln529, GenBank accession number ACP41105.1). The secondary structures of HIV-1 gp41, EBOV GP, and influenza virus HA were determined by circular dichroism spectroscopy (38). All the three proteins exhibited similar $\alpha$ helicity and $\beta$ strand to those in the UniProt database, confirming that the proteins have well-fold structures.

\section{Pseudotype virus production}

HEK293T cells $\left(5 \times 10^{5}\right)$ were seeded into six-well plates 24 hours before transfection. Then, HEK293T cells were cotransfected with $1.0 \mu \mathrm{g}$ of pNL4-3 plasmid, $12 \mathrm{ng}$ of pCMV3-EBOV GP, and $0.3 \mu \mathrm{g}$ of pAdvantage with the transfection reagent MegaTran 1.0 (OriGene) according to the manufacturer's instructions to produce EBOV GP pseudotyped HIV virions (HIV/EBOV GP). HEK293T cells were cotransfected with $1.0 \mu \mathrm{g}$ of pNL4-3 plasmid, $0.5 \mu \mathrm{g}$ of pcDNA4/ TO-HA, $0.5 \mu \mathrm{g}$ of pcDNA4/TO-NA, and $0.3 \mu \mathrm{g}$ of pAdvantage to produce pseudotyped IAV (IAVpp). HEK293T cells were cotransfected with $1.0 \mu \mathrm{g}$ of pNL4-3 plasmid, $0.7 \mu \mathrm{g}$ of pCMV-VSVG, and $0.3 \mu \mathrm{g}$ of pAdvantage to produce VSVG pseudotyped HIV virions (VSVpp). HEK293T cells were cotransfected with $1.0 \mu \mathrm{g}$ of pNL4-3 plasmid, $0.7 \mu \mathrm{g}$ of pCMV3-HIV Env, and $0.3 \mu \mathrm{g}$ of pAdvantage to produce HIV pseudotyped virions (HIVpp). HEK293T cells were cotransfected with $1.0 \mu \mathrm{g}$ of pNL4-3 plasmid, $0.5 \mu \mathrm{g}$ of pCMV3-HIV Env, and $0.3 \mu \mathrm{g}$ of pAdvantage to produce Marburg pseudotyped virions (MARVpp). The supernatants containing the pseudotyped viruses were collected twice (at 48 and 72 hours after transfection), combined, clarified by the removal of floating cells and cell debris with low-speed centrifugation, and filtered through a $0.45-\mu \mathrm{m}$ poresize filter (Nalgene). The culture supernatants containing pseudotyped viruses were either used immediately or flash frozen in aliquots and stored at $-80^{\circ} \mathrm{C}$ until use. Each aliquot was thawed only once for use in a single round of replication.

\section{Infection assay using pseudotyped viruses}

Compounds were tested using entry assays for HIV/EBOV GP, HIVpp, IAVpp, MARVpp, and VSVpp, as previously described $(7,8)$. Infections were performed in 96-well plates by adding diluted HIV/ EBOV GP, HIVpp, IAVpp, MARVpp, or VSVpp to $5 \times 10^{3}$ indicated cells per well in the presence and absence of the test compounds. The mixtures were then incubated for 72 hours at $37^{\circ} \mathrm{C}$ to screen the compound libraries. Luciferase activity, which reflected the number of pseudoparticles in the host cells, was measured 3 days after infection using the Bright-Glo reagent (Promega) and an FB15 luminometer (Berthold Detection Systems) according to the manufacturer's protocol. Test compounds were serially diluted to a final concentration of $1 \mu \mathrm{M}$ in $1 \%$ DMSO, and the maximum activity (100\% of the control) and background were derived from the control wells containing DMSO alone or from uninfected wells, respectively. The individual signals in each of the compound test wells were then divided by the average signals of the control values (wells lacking inhibitor) after subtracting the background, and the results were multiplied by 100 to determine the percent activity. The corresponding inhibition values were calculated by subtracting this value from 100. The specificity of the compounds for inhibiting HIV/EBOV GP was determined by evaluating the inhibition of IAVpp and VSVpp infection in parallel. Each sample was tested in duplicate, and all the experiments were repeated at least three times.

\section{Native EBOV infection inhibition assay under biosafety level 4 conditions}

Anti-native EBOV assays were conducted in biosafety level 4 at the U.S. Army Medical Research Institute of Infectious Diseases. HeLa and HFF cells were seeded into 96-well plates and exposed to native EBOV (Makona). HeLa cells were incubated with a serially diluted lead compound $(10,3.33,1.11,0.37,0.12,0.04,0.01,0.005$, and $0 \mu \mathrm{M})$ or $1 \%$ DMSO 2 hours before the addition of the virus $(\mathrm{MOI}=1.5)$. The viruses were added to the HFF cells at an MOI of 20, as measured in HeLa cells. The infection was stopped after 48 hours by fixing the cells with a formalin solution. Immunostaining was performed with an Alexa Fluor 488-conjugated anti-GP antibody to detect the infected cells. Images were acquired with the PE Opera confocal platform using a $10 \times$ objective and analyzed using Acapella software. Genedata software was used to calculate the percent infection from the GP staining data, and this value was then converted into percent inhibition. To evaluate the effect of lead compound on 
cell viability, CellTiter-Glo (Promega Corp., Madison, WI, USA) assay was conducted using a plate reader (Tecan Infinite M2000 PRO; Tecan Group Ltd., Mannedorf, Switzerland) (7). These experiments were independently repeated three times. Compound 3.47 was used as a positive control (12).

\section{Time-of-addition experiment}

The "time-of-addition" experiment was designed as previously described $(7,8,14)$ to determine the mechanism of action of the antiviral compounds, and the procedure is shown schematically in fig. S2. Briefly, A549 cells were seeded into 96-well plates at a density of $5 \times 10^{3}$ cells per well 24 hours before infection and incubated at $37^{\circ} \mathrm{C}$ with $5 \% \mathrm{CO}_{2}$. Then, the A549 cells were infected with HIV/ EBOV GP and treated with test compounds $(0.5 \mu \mathrm{M}$ Y11, $2 \mu \mathrm{M}$ Y18, or $2 \mu \mathrm{M}$ E-64d) at the various stages of viral entry. Duplicate wells were used for each time point, and control-infected cultures were treated with only a drug vehicle (DMSO). At 72 hours after infection, the cells were assayed, and the infectivity was measured as described above.

\section{Photoaffinity labeling and click chemistry}

Photoactivation and click chemistry were performed using a previously described method with some modifications (12,39). Briefly, cell lysates containing the packaged pseudotyped viruses were incubated with $50 \mu \mathrm{M}$ Y0, Y11, or Y12 or with DMSO for $30 \mathrm{~min}$ at room temperature. The lysates were then incubated with $5 \mu \mathrm{M}$ Y18 for an additional $30 \mathrm{~min}$ and exposed to UV light $(365 \mathrm{~nm}$ ) for $10 \mathrm{~min}$ on ice. Azido-immobilized agarose and $10 \mathrm{mM} \mathrm{L}$-ascorbic acid were then added sequentially. The azido-immobilized agarose was prepared by incubating excess biotin-azide (Invitrogen) with streptavidin (SA)-immobilized agarose (Invitrogen). The cycloaddition reaction (click chemistry) was initiated by adding $1 \mathrm{mM}$ $\mathrm{CuSO}_{4}$, and the samples were incubated overnight at $4^{\circ} \mathrm{C}$. The agarose was washed, and the captured target proteins were analyzed by Western blotting.

\section{Photoaffinity labeling and MS measurements}

Purified EBOV (strain Mayinga 1976, Zaire) GP protein was solubilized in ultrapure water to a final concentration of $1 \mathrm{mg} / \mathrm{ml}$. The protein was then incubated with $5 \mu \mathrm{M}$ Y18 or $1 \%$ DMSO for an additional $30 \mathrm{~min}$ and exposed to UV light $(365 \mathrm{~nm})$ for $10 \mathrm{~min}$ on ice. The probe-labeled protein was analyzed by peptide mapping. After an overnight trypsin digest at $37^{\circ} \mathrm{C}$, the reaction was quenched by the addition of trifluoroacetic acid (TFA). The tryptic peptides were resuspended and separated by $\mathrm{C} 18$ reversed-phase HPLC (RP-HPLC) (Easy-nLC II; Thermo Fisher Scientific, CA) with in-line UV detection. The fractions were collected, dried, and then resuspended in TFA. Each fraction was further separated on an analytical C18 column. The peptides were identified by MALDI (MS and MS/ MS) analysis. The photoaffinity labeling of HIV gp41 with Y20 and of influenza virus HA with Y21 and subsequent MS measurements were performed using the same procedure.

\section{Production and purification of the recombinant peptide ebolZN39IQ (N39)}

The design of the recombinant peptide eboIZN39IQ (N39) has been previously described (19). The optimized gene encoding eboIZN39IQ (N39) was synthesized by BGI (Beijing, China) and cloned into pET21a (+) vector between the Nde I and Xho I sites to generate
pET21-N39. The optimized gene sequence was CACCACCATCATCATCATATTGAAGGCCGCGGCCACATGGATAT CAAGAAAGAAATTGAGGCGATCAAGAAAGAGCAGGAAGCGATCAAGAAGAAGATCGAGGCGATCGAGAAAGAACTGCGTCAACTGGCAAACGAAACCACCCAAGCACTGCAACT GTTTCTGCGCGCAACCACCGAACTGCGTACCTTTAGCATCCTGAACCGCAAAGCGATCGATTTTCTGCTGCAGCGCATGAAGCAGATCGAAGACAAGATCGAAGAAATTGAGAGCAAGCAGAAGAAGATCGAGAACGAGATCGCGCGTATCAAAAAACTGATCGGCGAACGTTACTAAA. A 6× His tag was added at the $\mathrm{N}$ terminus for affinity purification, followed by the addition of a factor Xa cleavage site (IEGR). BL21 (DE3) was used as the host strain for eboIZN39IQ (N39) expression. The strain hosting pET21-N39 was inoculated into $2 \mathrm{ml}$ of Luria-Bertani (LB) medium containing ampicillin $(100 \mu \mathrm{g} / \mathrm{ml})$ and grown at $37^{\circ} \mathrm{C}$ while shaking at $220 \mathrm{rpm}$. The overnight Escherichia coli cultures were then diluted to an optical density (OD) of 0.2 in LB medium and incubated at $37^{\circ} \mathrm{C}$. When the culture ODs reached approximately $1.0\left(\mathrm{OD}_{600}\right)$, isopropyl- $\beta$-D-thiogalactopyranoside was added to a final concentration of $1 \mathrm{mM}$. After overnight induction at $30^{\circ} \mathrm{C}$, the cells were harvested by centrifugation and resuspended in His-Bind buffer [20 mM tris- $\mathrm{HCl}$ ( $\mathrm{pH} \mathrm{8.0),} 250 \mathrm{mM} \mathrm{NaCl}$, and $5 \mathrm{mM}$ imidazole]. The proteins were extracted by passing the cells twice through a microfluidizer at 1200 bar with cooling. The supernatant containing the recombinant peptide eboIZN39IQ (N39) was collected by centrifugation and mixed with Ni-NTA His-Bind resin (Novagen) for 2 hours at $4^{\circ} \mathrm{C}$. The unbound proteins were then removed by washing the resin with 10 volumes of wash buffer $[20 \mathrm{mM}$ tris- $\mathrm{HCl}(\mathrm{pH} 8.0)$, $250 \mathrm{mM} \mathrm{NaCl}$, and $20 \mathrm{mM}$ imidazole]. The resin-enriched eboIZN39IQ (N39) peptide was eluted with the same buffer, except that the concentration of imidazole was $500 \mathrm{mM}$. The purified peptide was dialyzed into $5 \%$ acetic acid, further purified by RP-HPLC on a C18 column (GE Healthcare), and then lyophilized. The peptide was then digested with factor Xa protease (New England Biolabs) according to the manufacturer's protocol. The digested peptide was dialyzed into $5 \%$ acetic acid, purified by HPLC, and then lyophilized again. The final peptide sequence was GHMDIKKEIEAIKKEQEAIKKKIEAIEKE $L R Q L A N E T T Q A L Q L F L R A T T E L$ RTFSILNRKAIDFLLQRMKQIEDKIEEIESKQKKIENEIARIKKLIGERY, where IZ $\mathrm{m}_{\mathrm{m}}$ and IQ are shown in bold and the EBOV $\mathrm{N}$-trimer in italics.

\section{Design and syntheses of eboC24 (C24) peptide}

The eboC24 (C24) peptide was designed and chemically synthesized by Abace Biology (Beijing, China). All peptides with $>95 \%$ purity were lyophilized, and their molecular weights were verified by LC-MS. The resulting eboC24 (C24) peptide sequence was Lys (N3)-IEPHDWTKNITDKIDQIIHDFVDK-NH ${ }_{2}$, and its molecular weight was $3074.45 \mathrm{Da}$.

\section{Surface plasmon resonance}

The interactions between the peptides and the compounds were analyzed with the Biacore T200 system (GE Healthcare, Uppsala, Sweden) at $25^{\circ} \mathrm{C}$. The recombinant eboIZN39IQ (N39) peptide was immobilized on a sensor chip (CM5) with the Amine Coupling Kit (GE Healthcare, Buckinghamshire, UK). The final levels of immobilized eboIZN39IQ (N39) were typically approximately 3000 response units (RU). The eboC24 (C24) peptide was conjugated to DIBO-biotin (Invitrogen) by click chemistry and then immobilized on an SA 
sensor chip, with final levels of approximately 600 RU. Various concentrations of the compounds were subsequently injected as analytes, and PBS-P [10 mM phosphate buffer containing $2.7 \mathrm{mM}$ $\mathrm{KCl}, 137 \mathrm{mM} \mathrm{NaCl}$, and $0.05 \%$ surfactant $\mathrm{P} 20$ (pH 4.5)] was used as the running buffer. For the binding studies, appropriate concentrations of the analytes were added to the running buffer at a flow rate of $30 \mu \mathrm{l} / \mathrm{min}$, a contact time of $120 \mathrm{~s}$, and a dissociation time of $60 \mathrm{~s}$, and the chip platforms were washed with running buffer and 50\% DMSO. The data were analyzed with Biacore evaluation software (T200 version 1.0), and the curve was fitted with a 1:1 binding model.

The eboIZN39IQ (N39) peptide was immobilized on a CM5 sensor chip at approximately $3000 \mathrm{RU}$ to explore whether Y11eboC24 binding had any effect on the interactions between the eboC24 and eboIZN39IQ (N39) peptides in the SPR experiments. EboC24 concentrations of $25,12.5,6.25,3.12,1.56,0.78,0.39$, and $0 \mu \mathrm{M}$ were passed through the chip at a flow rate of $10 \mu \mathrm{l} / \mathrm{min}$, a contact time of $60 \mathrm{~s}$, and a dissociation time of $600 \mathrm{~s}$ in the absence or presence of Y11 or other compounds. The N39 peptide-SA chip was regenerated with glycine $(\mathrm{pH} 2.5)$. The data were analyzed with Biacore evaluation software (T200 Version 1.0), and the curve was fitted with a 1:1 binding model.

\section{Mapping the HR2 domain using amino acid substitutions}

We performed mutagenesis on the HR2 domain and tested the activity of the lead compounds against the EBOV mutants to identify which HR2 residues interacted with the lead compounds. Briefly, each $\mathrm{HR} 2$ residue (residues 610 to 636) was separately replaced with alanine (A) by site-directed mutagenesis (Agilent Technologies) and confirmed by sequencing (BGI, Beijing, China). The resulting plasmids were used to package the EBOV mutants. The infectivity of the EBOV mutants and the antiviral activity against them were evaluated as described above. The HR2 domain from influenza virus was mapped in the same manner by using amino acid substitutions.

\section{Nuclear magnetic resonance}

The HR2 peptide samples used for NMR spectroscopy were prepared by dissolving an appropriate amount of peptide in $0.5 \mathrm{ml}$ of solution $\left(\mathrm{H}_{2} \mathrm{O} / \mathrm{D}_{2} \mathrm{O}=9: 1\right)$ to obtain a peptide concentration of $1.0 \mathrm{mM}$. The peptide-Y11 complex was prepared by the same procedure by mixing the two components at a ligand/peptide ratio of 1:1. The NMR spectra were recorded on a Bruker DRX-600 spectrometer equipped with a cryoprobe. 1D NMR spectra were recorded in Fourier mode with quadrupole detection. The TOCSY and NOESY (nuclear Overhauser effect spectroscopy) experiments were performed in a phase-sensitive mode using quadrupole detection in $\omega 1$ with a time-dependent phase increase in the initial pulse. The data block sizes were 2048 addresses in $t_{2}$ and 512 equidistant $t_{1}$ values. Before the Fourier transformation, the time domain data matrices were multiplied by shifting the sine functions in both dimensions. The TOCSY experiments were performed at $298 \mathrm{~K}$ with a mixing time of $200 \mathrm{~ms}$. The NOESY experiments were performed at $298 \mathrm{~K}$ with a mixing time of $300 \mathrm{~ms}$. The NMR spectra were analyzed using MestReNova software.

\section{Docking simulation}

The Y11 and Y18 compounds were docked to the EBOV GP protein by AutoDock 4.2. The structural template of EBOV GP (Protein Data Bank: 5JQ3) was obtained from the RCSB Protein Data Bank (www. rcsb.org/pdb/home/home.do). Grid points covering EBOV HR2 were generated with AutoDock Tools to perform blind docking experiments. The box size was $80 \times 80 \times 80$ points with a standard spacing of $0.375 \AA$. Docking simulations for the compounds were performed using the Lamarckian genetic algorithm (GA) and a protocol with 50 GA runs, an initial population of 300 randomly placed individuals, a maximum number of 25 million energy evaluations, a mutation rate of 0.02 , and a crossover rate of 0.80 . The resulting conformations, which differed by less than $2.0 \AA$ in the positional root mean square deviation, were clustered together. The default values were used for all other parameters. All relevant torsion angles were treated as rotatable during the docking process to allow searching of the conformational space. After AutoGrid and AutoDock were run, the possible positions of the compounds on EBOV GP were obtained. The same procedure was conducted for the docking simulations of Y19 and Y20 with HIV-1 gp160 (PDB: 4TVP) and of Y3 and Y21 with influenza virus HA (PDB: 3LZG).

\section{Production of polyclonal antibodies against the HR2 peptide (KIDQIIHDF)}

A polyclonal antibody was generated by immunizing New Zealand rabbits with the synthesized HR2 peptide mariculture keyhole limpet hemocyanin (mcKLH) conjugate. The maleimide-activated mcKLH carrier protein (Pierce) was reconstituted by adding $\mathrm{dH}_{2} \mathrm{O}$ to Hypovial to yield a solution of $10 \mathrm{mg} / \mathrm{ml}$. A mixture of $2 \mathrm{mg}$ of peptide and $2 \mathrm{mg}$ of activated mcKLH was used, and the mcKLH formed a suspension, not a solution, that was typically opaque to whitish blue in color. The suspension was not solubilized by vortexing or heating, as these treatments would cause mcKLH to further precipitate from solution. Next, $2 \mathrm{mg}$ of sulfhydryl-containing hapten was dissolved in $200 \mu \mathrm{l}$ of $\mathrm{dH}_{2} \mathrm{O}$. The peptide and activated mcKLH solutions were then immediately mixed, and the mixture was allowed to react for 2 hours at room temperature. The conjugate was purified by dialysis against PBS buffer. For the first immunization, the purified peptide-mcKLH conjugate $(100 \mu \mathrm{g})$ in PBS $(1 \mathrm{ml})$ was mixed with an equal volume of Freund's complete adjuvant (Bio Basic Inc.) to form a stable emulsion. One rabbit was subcutaneously injected at two to four different sites. Three booster injections were administered at 20 -day intervals using $100 \mu \mathrm{g}$ of the purified peptide-mcKLH conjugate with incomplete Freund's adjuvant. Blood samples (0.5 to $1 \mathrm{ml})$ were collected before each injection, and bleeding $(30 \mathrm{ml})$ was performed 10 days after the last booster to evaluate the immune response. Polyclonal antibodies (IgGs) were purified from rabbit serum by affinity chromatography on a 5-ml HiTrap protein A column (GE Healthcare) according to the manufacturer's instructions. Binding was performed in $0.02 \mathrm{M}$ sodium phosphate ( $\mathrm{pH} 7.0$ ), and elution was performed in $0.1 \mathrm{M}$ citric acid ( $\mathrm{pH} 3.0$ ). The eluted IgGs were collected, immediately neutralized to physiological $\mathrm{pH}$ by adding $1 \mathrm{M}$ tris- $\mathrm{HCl}$ buffer ( $\mathrm{pH} 9.0$ ), and then concentrated to $1 \mathrm{mg} / \mathrm{ml}$ by using $30-\mathrm{kDa}$ molecular weight cutoff concentrators. Next, an $\mathrm{N}$-hydroxysuccinimide (NHS)-activated Sepharose column (GE Healthcare) was used for affinity purification of the HR2-specific IgGs from the total rabbit antibodies. Concentrated HR2 peptide (20 mg) in coupling buffer [0.2 $\mathrm{M} \mathrm{NaHCO}_{3}$ and $\left.0.5 \mathrm{M} \mathrm{NaCl}(\mathrm{pH} 8.3)\right]$ was applied to the NHS-activated column and allowed to bind for 2 hours at room temperature. Excess uncoupled, activated NHS groups were deactivated by sequential washing with two buffers: $0.5 \mathrm{M}$ ethanolamine and $0.5 \mathrm{M} \mathrm{NaCl}(\mathrm{pH} 8.3)$, followed by $0.1 \mathrm{M}$ sodium acetate and $0.5 \mathrm{M} \mathrm{NaCl}(\mathrm{pH} 4.0)$. The IgGs purified from rabbit serum using protein A were passed through the HR2 column. Unbound 
IgGs were removed by wash buffer [0.05 $\mathrm{M}$ sodium phosphate and $0.15 \mathrm{M} \mathrm{NaCl}$ (pH 7.0)] to obtain pure HR2-specific IgGs, which were eluted with three- to five-column volumes of $0.1 \mathrm{M}$ citric acid buffer and then neutralized with $1 \mathrm{M}$ tris- $\mathrm{HCl}$ buffer ( $\mathrm{pH}$ 9.0). $\mathrm{Pu}-$ rified HR2-specific IgG samples were buffer exchanged with $0.05 \mathrm{M}$ sodium phosphate buffer ( $\mathrm{pH} 7.0$ ) and then concentrated to $1 \mathrm{mg} / \mathrm{ml}$. All animal experiments were performed in accordance with the guidelines of the Institutional Animal Care and Use Committee of Peking University.

\section{Detection of the HR2 peptide (KIDQIIHDF)-specific polyclonal antibody}

An indirect enzyme-linked immunosorbent assay (ELISA) assay (40) was used to analyze sera and purified IgG. The HR2 peptide antigen was diluted in carbonate coating buffer, and then, $100 \mu$ of this solution was added to the wells of an ELISA microplate and incubated overnight at $4^{\circ} \mathrm{C}$. After the plate was washed three times with PBS containing $0.05 \%$ Tween 20 to remove unbound antigen, $150 \mu$ of blocking buffer ( $3 \%$ bovine serum albumin in wash buffer) was applied to all wells for 1 hour at $37^{\circ} \mathrm{C}$. After removing the blocking buffer, $100 \mu \mathrm{l}$ of rabbit sera or IgGs diluted in $1 \%$ blocking buffer was added to each well, and the plate was incubated for an additional 1 hour at room temperature. After three washes, HRP-conjugated goat anti-rabbit IgG was diluted (1:5000) in 1\% blocking buffer, added to each well (100 $\mu$ l per well), and allowed to bind to the captured rabbit IgG for 1 hour at room temperature. After five additional washes, the bound conjugate was detected with $50 \mu$ of 3,3',5,5'-tetramethylbenzidine (Sigma-Aldrich) substrate, and the reaction was stopped after $8 \mathrm{~min}$ by the addition of $50 \mu \mathrm{l}$ of $1 \mathrm{M}$ $\mathrm{H}_{2} \mathrm{SO}_{4}$. The spectroscopic absorbance of each well was measured by an automated plate reader at a wavelength of $450 \mathrm{~nm}$.

\section{SUPPLEMENTARY MATERIALS}

Supplementary material for this article is available at http://advances.sciencemag.org/cgi/ content/full/4/11/eaau8408/DC1

Supplementary Materials and Methods

Fig. S1. Discovery of pentacyclic triterpene glycoconjugates as EBOV entry inhibitors.

Fig. S2. Time-of-addition experiments to clarify the stage at which lead compounds blocked EBOV entry.

Fig. S3. Characterization of the affinity of triterpenoid compounds to N-terminal HR1, C-terminal HR2, and their effect on HR1-HR2 interaction.

Fig. S4. Characterization of the spatial interactions within the triterpenoid-HR2 complex according to NMR spectra.

Fig. S5. Docking simulation and biological mapping supporting a structural model of the triterpenoid lead compound-HR2 complex.

Fig. S6. Characterization of the affinity of triterpenoid compounds Y19 and Y20 to HIV HR2 and HR1 and their effect on HR1-HR2 interactions.

Fig. S7. Identification of HR2 in influenza HA2 as the domain targeted by the triterpenoid leads.

Fig. S8. Production and characterization of the HR2 peptide (KIDQIIHDF)-specific polyclonal antibody.

Fig. S9. The structure-activity relationship of triterpenoids against viruses according to our study.

Table S1. Broad antiviral spectra of the tested compounds against various EBOV subtypes and MARV.

Movie S1. The EBOV-host membrane fusion via a trimer-of-hairpins.

Movie S2. Pentacyclic triterpene lead compounds inhibit virus-host membrane fusion by targeting the HR2 of virus envelope protein.

\section{REFERENCES AND NOTES}

1. S. C. Harrison, Viral membrane fusion. Nat. Struct. Mol. Biol. 15, 690-698 (2008).

2. T. G. Morrison, Structure and function of a paramyxovirus fusion protein. Biochim. Biophys. Acta 1614, 73-84 (2003).
3. J. M. White, S. E. Delos, M. Brecher, K. Schornberg, Structures and mechanisms of viral membrane fusion proteins: Multiple variations on a common theme. Crit. Rev. Biochem. Mol. Biol. 43, 189-219 (2008).

4. V. Domingo, J. F. Arteaga, J. F. Quilez del Moral, A. F. Barrero, Unusually cyclized triterpenes: Occurrence, biosynthesis and chemical synthesis. Nat. Prod. Rep. 26, 115-134 (2009).

5. P. F. Smith, A. Ogundele, A. Forrest, J. Wilton, K. Salzwedel, J. Doto, G. P. Allaway, D. E. Martin, Phase I and II study of the safety, virologic effect, and pharmacokinetics/ pharmacodynamics of single-dose 3-o-(3,'3'-dimethylsuccinyl)betulinic acid (bevirimat) against human immunodeficiency virus infection. Antimicrob. Agents Chemother. 51, 3574-3581 (2007).

6. J. F. Mayaux, A. Bousseau, R. Pauwels, T. Huet, Y. Hénin, N. Dereu, M. Evers, F. Soler, C. Poujade, E. Declercq, Triterpene derivatives that block entry of humanimmunodeficiency-virus type-1 into cells. Proc. Natl. Acad. Sci. U.S.A. 91, 3564-3568 (1994).

7. M. R. Yu, L. L. Si, Y. F. Wang, Y. M. Wu, F. Yu, P. X. Jiao, Y. Y. Shi, H. Wang, S. L. Xiao, G. Fu, K. Tian, Y. T. Wang, Z. H. Guo, X. S. Ye, L. H. Zhang, D. M. Zhou, Discovery of pentacyclic triterpenoids as potential entry inhibitors of influenza viruses. J. Med. Chem. 57, 10058-10071 (2014).

8. F. Yu, Q. Wang, Z. Zhang, Y. Y. Peng, Y. Y. Qiu, Y. Y. Shi, Y. X. Zheng, S. L. Xiao, H. Wang, X. X. Huang, L. Y. Zhu, K. B. Chen, C. K. Zhao, C. L. Zhang, M. R. Yu, D. A. Sun, L. H. Zhang, D. M. Zhou, Development of oleanane-type triterpenes as a new class of HCV entry inhibitors. J. Med. Chem. 56, 4300-4319 (2013).

9. B. Labrosse, C. Treboute, M. Alizon, Sensitivity to a nonpeptidic compound (RPR103611) blocking human immunodeficiency virus type 1 Env-mediated fusion depends on sequence and accessibility of the gp41 loop region. J. Virol. 74, 2142-2150 (2000).

10. V. N. Malashkevich, B. J. Schneider, M. L. McNally, M. A. Milhollen, J. X. Pang, P. S. Kim, Core structure of the envelope glycoprotein GP2 from Ebola virus at 1.9-angstrom resolution. Proc. Natl. Acad. Sci. U.S.A. 96, 2662-2667 (1999).

11. J. M. White, K. L. Schornberg, A new player in the puzzle of filovirus entry. Nat. Rev. Microbiol. 10, 317-322 (2012).

12. M. Côté, J. Misasi, T. Ren, A. Bruchez, K. Lee, C. M. Filone, L. Hensley, Q. Li, D. Ory, K. Chandran, J. Cunningham, Small molecule inhibitors reveal Niemann-Pick C1 is essential for Ebola virus infection. Nature 477, 344-348 (2011).

13. E. Picazo, F. Giordanetto, Small molecule inhibitors of ebola virus infection. Drug Discov. Today 20, 277-286 (2015).

14. A. Basu, B. Li, D. M. Mills, R. G. Panchal, S. C. Cardinale, M. M. Butler, N. P. Peet, H. Majgier-Baranowska, J. D. Williams, I. Patel, D. T. Moir, S. Bavari, R. Ray, M. R. Farzan, L. Rong, T. L. Bowlin, Identification of a small-molecule entry inhibitor for filoviruses. J. Virol. 85, 3106-3119 (2011).

15. J. Das, Aliphatic diazirines as photoaffinity probes for proteins: Recent developments. Chem. Rev. 111, 4405-4417 (2011).

16. P. Thirumurugan, D. Matosiuk, K. Jozwiak, Click chemistry for drug development and diverse chemical-biology applications. Chem. Rev. 113, 4905-4979 (2013).

17. Y. Zhao, J. Ren, K. Harlos, D. M. Jones, A. Zeltina, T. A. Bowden, S. Padilla-Parra, E. E. Fry, D. I. Stuart, Toremifene interacts with and destabilizes the Ebola virus glycoprotein Nature 535, 169-172 (2016).

18. J. E. Lee, M. L. Fusco, A. J. Hessell, W. B. Oswald, D. R. Burton, E. O. Saphire, Structure of the Ebola virus glycoprotein bound to an antibody from a human survivor. Nature 454, 177-182 (2008).

19. T. R. Clinton, M. T. Weinstock, M. T. Jacobsen, N. Szabo-Fresnais, M. J. Pandya, F. G. Whitby, A. S. Herbert, L. I. Prugar, R. McKinnon, C. P. Hill, B. D. Welch, J. M. Dye, D. M. Eckert, M. S. Kay, Design and characterization of ebolavirus GP prehairpin intermediate mimics as drug targets. Protein Sci. 24, 446-463 (2015).

20. L. N. Makley, K. A. McMenimen, B. T. DeVree, J. W. Goldman, B. N. McGlasson, P. Rajagopal, B. M. Dunyak, T. J. McQuade, A. D. Thompson, R. Sunahara, R. E. Klevit, U. P. Andley, J. E. Gestwicki, Pharmacological chaperone for $\alpha$-crystallin partially restores transparency in cataract models. Science 350, 674-677 (2015).

21. S. Watanabe, A. Takada, T. Watanabe, H. Ito, H. Kida, Y. Kawaoka, Functional importance of the coiled-coil of the Ebola virus glycoprotein. J. Virol. 74, 10194-10201 (2000).

22. L. W. McGinnes, T. Sergel, H. Chen, L. Hamo, S. Schwertz, D. Li, T. G. Morrison, Mutational analysis of the membrane proximal heptad repeat of the newcastle disease virus fusion protein. Virology 289, 343-352 (2001).

23. S. T. Yang, A. J. B. Kreutzberger, V. Kiessling, B. K. Ganser-Pornillos, J. M. White, L. K. Tamm, HIV virions sense plasma membrane heterogeneity for cell entry. Sci. Adv. 3, e1700338 (2017).

24. F. Soler, C. Poujade, M. Evers, J. C. Carry, Y. Hénin, A. Bousseau, T. Huet, R. Pauwels, E. DeClercq, J. F. Mayaux, J. B. LePecq, N. Dereu, Betulinic acid derivatives: A new class of specific inhibitors of human immunodeficiency virus type 1 entry. J. Med. Chem. 39, 1069-1083 (1996).

25. M. Pancera, T. Zhou, A. Druz, I. S. Georgiev, C. Soto, J. Gorman, J. Huang, P. Acharya, G. Y. Chuang, G. Ofek, G. B. Stewart-Jones, J. Stuckey, R. T. Bailer, M. G. Joyce, M. K. Louder, N. Tumba, Y. Yang, B. Zhang, M. S. Cohen, B. F. Haynes, J. R. Mascola, L. Morris, J. B. Munro, 
S. C. Blanchard, W. Mothes, M. Connors, P. D. Kwong, Structure and immune recognition of trimeric pre-fusion HIV-1 Env. Nature 514, 455-461 (2014).

26. D. M. Eckert, P. S. Kim, Design of potent inhibitors of HIV-1 entry from the gp41 N-peptide region. Proc. Natl. Acad. Sci. U.S.A. 98, 11187-11192 (2001).

27. P. A. Bullough, F. M. Hughson, J. J. Skehel, D. C. Wiley, Structure of influenza haemagglutinin at the $\mathrm{pH}$ of membrane fusion. Nature 371, 37-43 (1994).

28. R. Xu, D. C. Ekiert, J. C. Krause, R. Hai, J. E. Crowe Jr., I. A. Wilson, Structural basis of preexisting immunity to the $2009 \mathrm{H} 1 \mathrm{~N} 1$ pandemic influenza virus. Science $\mathbf{3 2 8}$, 357-360 (2010).

29. P. Chambers, C. R. Pringle, A. J. Easton, Heptad repeat sequences are located adjacent to hydrophobic regions in several types of virus fusion glycoproteins. J. Gen. Virol. 71 (Pt. 12) 3075-3080 (1990).

30. D. C. Chan, D. Fass, J. M. Berger, P. S. Kim, Core structure of gp41 from the HIV envelope glycoprotein. Cell 89, 263-273 (1997).

31. S. B. Joshi, R. E. Dutch, R. A. Lamb, A core trimer of the paramyxovirus fusion protein: Parallels to influenza virus hemagglutinin and HIV-1 gp41. Virology 248, 20-34 (1998).

32. J. M. Kilby, S. Hopkins, T. M. Venetta, B. DiMassimo, G. A. Cloud, J. Y. Lee, L. Alldredge, E. Hunter, D. Lambert, D. Bolognesi, T. Matthews, M. R. Johnson, M. A. Nowak, G. M. Shaw, M. S. Saag, Potent suppression of HIV-1 replication in humans by T-20, a peptide inhibitor of gp41-mediated virus entry. Nat. Med. 4, 1302-1307 (1998).

33. J. K. Young, D. Li, M. C. Abramowitz, T. G. Morrison, Interaction of peptides with sequences from the Newcastle disease virus fusion protein heptad repeat regions. J. Virol. 73, 5945-5956 (1999).

34. M. Gochin, G. Zhou, Amphipathic properties of HIV-1 gp41 fusion inhibitors. Curr. Top. Med. Chem. 11, 3022-3032 (2011).

35. P. Forterre, D. Prangishvili, The origin of viruses. Res. Microbiol. 160, 466-472 (2009).

36. A. Nasir, G. Caetano-Anollés, A phylogenomic data-driven exploration of viral origins and evolution. Sci. Adv. 1, e1500527 (2015).

37. S. Alakurtti, T. Makela, S. Koskimies, J. Yli-Kauhaluoma, Pharmacological properties of the ubiquitous natural product betulin. Eur. J. Pharm. Sci. 29, 1-13 (2006).

38. N. A. Lakomek, J. D. Kaufman, S. J. Stahl, J. M. Louis, A. Grishaev, P. T. Wingfield, A. Bax Internal dynamics of the homotrimeric HIV-1 viral coat protein gp41 on multiple time scales. Angew. Chem. Int. Ed. Engl. 52, 3911-3915 (2013).

39. H. Shi, C. J. Zhang, G. Y. Chen, S. Q. Yao, Cell-based proteome profiling of potential dasatinib targets by use of affinity-based probes. J. Am. Chem. Soc. 134 3001-3014 (2012).
40. L. L. Si, H. Xu, X. Y. Zhou, Z. W. Zhang, Z. Y. Tian, Y. Wang, Y. M. Wu, B. Zhang, Z. L. Niu, C. L. Zhang, G. Fu, S. L. Xiao, Q. Xia, L. H. Zhang, D. M. Zhou, Generation of influenza A viruses as live but replication-incompetent virus vaccines. Science 354, 1170-1173 (2016).

Acknowledgments: We thank J. M. Cunningham from Harvard Medical School for his assistance with the anti-native EBOV screening work. We thank Z. Liu from Peking University for his assistance with the docking simulation. We thank X. Liu from Institute of Biophysics, Chinese Academy of Sciences, for his assistance with the NMR experiments. Funding: This work was supported by the National Natural Science Foundation of China (grant nos. $81821004,91753202,21572015,81530090,81373271,21877007$, and 81361168002) and the National Basic Research Program of China (grant no. 2016YFA0501500). Author contributions: D.Z. and L.S. conceived the study, designed experiments, interpreted data, and wrote the manuscript. L.Z. revised the manuscript. L.S. performed most of the experiments and interpreted data except those noted. K.M., Z.T., J.S., H.L., Z.Z., V.S., H.L., G.F., Q.X., and S.X. provided assistance on part of the experiments. In particular, K.M. and J.S. conducted the NMR experiment and analyzed the NMR data. Competing interests: D.Z., L.S., Z.Z., K.M., S.X., and L.Z. are inventors on a patent application issued by the state intellectual property office of the People's Republic of China and held by Peking University that covers the application of triterpenoids as EBOV and MARV inhibitors (no. 201610248269.0; filed on 20 April 2016). The authors declare no other competing interests. Data and materials availability: The genome sequences have been deposited in GenBank under the accession numbers U23187.1 (Zaire EBOV GP), YP_138523.1 (Sudan EBOV GP), ACI28624.1 (Bundibugyo EBOV GP), ACI28632.1 (Ivory Coast EBOV GP), AAC54891.1 (Reston EBOV GP), YP_001531156.1 (Musoke Marburg GP), and M27323.1 (HIV-1, group M, subtype D, strain NDK). Data generated by this study are included in the main text and the Supplementary Materials and are available from the corresponding author upon request.

Submitted 19 July 2018

Accepted 24 October 2018

Published 21 November 2018

10.1126/sciadv.aau8408

Citation: L. Si, K. Meng, Z. Tian, J. Sun, H. Li, Z. Zhang, V. Soloveva, H. Li, G. Fu, Q. Xia, S. Xiao, L. Zhang, D. Zhou, Triterpenoids manipulate a broad range of virus-host fusion via wrapping the HR2 domain prevalent in viral envelopes. Sci. Adv. 4, eaau8408 (2018). 